\title{
Asymptotics of the Spectrum and the Selberg Zeta Function on the Space of Riemann Surfaces
}

\author{
Scott A. Wolpert* \\ Department of Mathematics, University of Maryland, College Park, MD 20742, USA
}

\begin{abstract}
Let $Z(s, R)$ be the Selberg zeta function of a compact Riemann surface $R$. We study the behavior of $Z(s, R)$ as $R$ tends to infinity in the moduli space of stable curves. The main result is an estimate for $Z(s, R)$ valid for $s$ in a neighborhood, depending only on the genus, of $s=1$. Our analysis gives an alternate proof of the Belavin-Knizhnik double pole result, [5].
\end{abstract}

\section{Table of Contents}

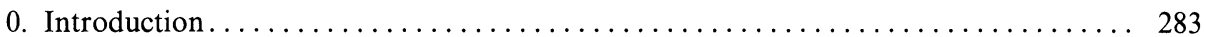

1. A Discussion of the Results ................................... 287

2. Collars and the Degeneration Paradigm ........................... 289

3. Bounding the Eigenvalue Spectrum of a Surface by the Spectrum of the Collars............................................ 296

4. Estimates for the Trace of the Heat Kernel ...................... 300

5. Estimates for the Selberg Zeta Function ........................ 306

6. Asymptotics of the Bosonic Polyakov $(d=26)$ Integral $\ldots \ldots \ldots \ldots \ldots \ldots \ldots \ldots \ldots$

\section{Introduction}

The functional determinant of the Laplacian appears in the Ray-Singer definition of analytic torsion $[13,28]$ and more recently in the Polyakov quantization of string theory $[4,9,16,26]$. An open problem is the behavior of spectral invariants for a degenerating family of metrics. We shall consider the question for compact surfaces with hyperbolic (constant curvature -1) metrics. For this case D'Hoker-Phong [10], Kierlanczyk [21] and Sarnak [29] have collectively evaluated the determinant of the Laplacian on functions and forms in terms of the Selberg zeta function $Z(s)$. $\mathrm{Up}$ to an elementary factor the determinant on functions (the 0 -mode is deleted) is $Z^{\prime}(1)$. More generally if $\Delta_{n}^{+}$is the $\partial$-Laplacian, and $\Delta_{n}^{-}$the $\bar{\partial}$-Laplacian, on tensors

* Partially supported by the National Science Foundation and the Institute for Physical Science and Technology, University of Maryland, College Park, MD, USA 
$f(z) d z^{n}$, then D'Hoker-Phong find constants $c_{n}$ such that $\operatorname{det} \Delta_{n}^{+}=e^{-c_{n} \chi} Z(n+1)$ and $\operatorname{det}^{\prime} \Delta_{n}^{-}=e^{-c_{n-1} \chi} Z(n)$, where $\chi$ is the Euler characteristic of the surface, [10]. Recall that for $\operatorname{Re} s>1, Z$ is calculated by a convergent formula from the length spectrum and for all $s$ from the eigenvalue spectrum, [18, 38 (Chaps. 1 and 2)].

One way of looking at the problem of spectral degeneration is to estimate $\theta(t)=\Sigma e^{-\lambda_{n} t}$, the trace of the heat kernel. As a brief indication of our results we find for $m$ lengths $\{\ell\}_{j=1}^{m}$ tending to zero that $Z^{\prime}(1)$ is bounded above and below by $\prod_{j=1}^{m} \exp \left(-\pi^{2} / 3 \ell_{j}\right) \ell_{j}^{-1} \prod_{0<\lambda_{n}<\frac{1}{4}} \lambda_{n}$ and $Z(2)$ by $\prod_{j=1}^{m} \exp \left(-\pi^{2} / 3 \ell_{j}\right) \ell_{j}^{-3}$. D'HokerPhong have found that the Polyakov integrand is

$$
c Z^{\prime}(1)^{-13} Z(2) d \mathrm{~W}-\mathrm{P},
$$

for a constant $c$ depending on the genus and the Weil-Petersson measure. By our analysis $Z^{\prime}(1)^{-13} Z(2)$ is comparable to

$$
\prod_{j=1}^{m} \exp \left(4 \pi^{2} / \ell_{j}\right) \ell_{j}^{10}\left(\prod_{0<\lambda_{n}<\frac{1}{4}} \lambda_{n}\right)^{-13} .
$$

The small eigenvalues are already known to be comparable to the short geodesic lengths (see Theorem 3.1) [11,30]. Now in Sect. 2.5 we show that $\exp \left(-2 \pi^{2} / \ell_{j}\right) \approx\left|\tau_{j}\right|$, where $\tau_{j}$ is a holomorphic parameter for the deformation. Thus the product $Z^{\prime}(1)^{-13} Z(2)$ has the order of magnitude of $\prod_{j=1}^{m}\left|\tau_{j}\right|^{-2}$ $\times\left(\log \left|\tau_{j}\right|\right.$ terms $)$. Masur has already observed that the Weil-Petersson measure is comparable to $\prod_{j=1}^{m}\left|d \tau_{j}\right|^{2} /\left|\tau_{j}\right|^{2}\left(\log 1 /\left|\tau_{j}\right|\right)^{3},[22]$. In conclusion the Polyakov integrand has the order of magnitude of the absolute square of a holomorphic function with a second order pole at infinity; this is in complete agreement with the BelavinKnizhnik result, [5]. Unfortunately our estimate relating the le ${ }_{1}$ gths $\ell_{j}$ and the holomorphic coordinates $\tau_{j}$ is not sufficient to find the contribution of the lower order term $\prod_{j=1}^{m} \ell_{j}^{10} \prod_{0<\lambda_{n}<\frac{1}{4}} \lambda_{n}^{-13}$ after the change of variables.

The analysis of the degeneration of $\theta(t)$ consists of two parts. The first is to obtain estimates for the degeneration of a hyperbolic metric and the second to estimate the effect on the spectrum. Before giving our precise results (see Chap. 1 for a summary) we would like to discuss the underlying ideas.

The theory of degeneration of hyperbolic metrics was developed by Bers [6] and Earle-Marden [12]. A brief exposition appears in Chap. 2. The first step is to study the degeneration of the hyperbolic metric for the annulus $\{|\tau|<|z|<1\}$ in the $z$-plane. For this basic case the hyperbolic metric, the Teichmüller space and even the Weil-Petersson metric can be computed explicitly (see Sects. 2.2 and 2.3). In particular the length of the core geodesic is $\ell=2 \pi^{2} /(\log 1 /|\tau|)$. The collar $C(\ell)$ about a core geodesic of length $\ell$ is defined to be the subannulus of points within distance $\log 2 / \ell$. It is a fundamental principle that the degeneration of a hyperbolic metric for any surface of finite topological type localizes into collar neighborhoods about the short geodesics. Thus the example of the annulus is actually a model for the general case. We formulate and prove precise versions of this principle. In particular by the 
simple construction of an associated surface we see that the geometry of the complement of the collars is uniformly bounded. The introduction of the associated surface is a key point of our approach. As a consequence we note that the functional determinant for the Dirichlet problem of the collar complement is bounded above and below in terms of the genus.

An important part of the degeneration theory of surfaces is the description of the compactification divisor $\mathscr{D} \subset \overline{\mathscr{M}}_{g}$ for the moduli space. We consider a simple method for estimating the asymptotics as $\varepsilon \rightarrow 0$ of integrals over the complement of an $\varepsilon$-neighborhood of $\mathscr{D}$. Provided the integrand is estimated above and below (to the same order) by the lengths of short geodesics, the method gives upper and lower bounds of the same order for the integral. Perhaps the method can be of further use in the future. In the meanwhile it is immediate at least, that the bosonic $(d=26)$ Polyakov integral is infinite.

The idea for estimating $Z(s)$, $s$ real, $s>\frac{1}{2}$ is suggested by McKean's formula

$$
\frac{Z^{\prime}(s)}{Z(s)}=(2 s-1) \int_{0}^{\infty} e^{-\xi t}(\theta(t)-k(t) \text { Area }(R)) d t
$$

for $\operatorname{Re} \xi>0, \xi=s^{2}-s$ and $k(t)$ the elementary solution of the heat equation, evaluated at the source, [24]. Essentially this is a Laplace transform in the variable $\xi$. The poles in the range $-\frac{1}{4}<\xi \leqq 0$ are the transform of the terms $e^{-\lambda t}, 0 \leqq \lambda<\frac{1}{4}$ from the heat trace. Thus quite simply if we remove the contribution of the small eigenvalues and define $\theta_{*}(t)=\sum_{\lambda_{n} \geqq \frac{1}{4}} e^{-\lambda_{n} t}$, replace $\theta$ by $\theta_{*}$ in McKean's integral then the left-hand side becomes $\frac{Z^{\prime}(s)}{Z(s)}-\sum_{0 \leqq \lambda_{n}<\frac{1}{4}} \frac{2 s-1}{s^{2}-s+\lambda_{n}}$ and for the right-hand side the integrand is now $O\left(e^{-t / 4}\right)$ for large $t$, thus the integral is convergent for $\operatorname{Re} \xi>-\frac{1}{4}$. This is our method for evaluating $Z(s)$ for $s$ real, $s>\frac{1}{2}$. The behavior of the small eigenvalues is already known $[11,30]$ (see Sect. 3.2) and as we shall see represents a separate phenomenon. Thus quite naturally the problem is reduced to considering $\theta_{*}(t)$.

Actually it is not so easy to obtain estimates of $\frac{Z^{\prime}(s)}{Z(s)}, s$ real, fixed, $s>\frac{1}{2}$ from McKean's formula. Recall that for small time $k(t)=\frac{1}{4 \pi t}+O(1)$ and $\theta_{*}(t)=\frac{g-1}{t}$ $+O(1)$, thus to even obtain a finite estimate for $\frac{Z^{\prime}(s)}{Z(s)}$ the upper and lower bounds for $\theta_{*}(t), t$ small, must each be of the form $\left(\frac{g-1}{t}+\right.$ integrable function $)$. In as much as the small $t$ behavior of $\theta_{*}(t)$ is governed by the asymptotics of the spectrum, the corresponding eigenvalue estimates must actually be asymptotically sharp. Furthermore to obtain a finite bound for $\frac{Z^{\prime}(s)}{Z(s)}, \frac{1}{2}<s \leqq 1$ the estimates for $\theta_{*}(t)$ must be of the form $O\left(e^{-t / 4}\right)$ for $t$ large.

Our plan is to use the uniformly bounded geometry of the collar complement and the standard eigenvalue comparison theorems to reduce the problem to estimating $\theta_{*}(t, D C(\ell))$ and $\theta_{*}(t, N C(\ell))$, the traces with small eigenvalues excluded, for the Dirichlet and Neumann problems of a collar. A collar has a 
rotational symmetry which can be used (separation of variables) to further reduce the considerations to a Sturm-Liouville (1-dimensional) problem. We find the estimate

$$
\theta_{*}(t, D C(\ell)) \leqq \theta_{*}(t, N C(\ell)) \leqq \theta_{*}(t, D C(\ell))+\theta_{1}(t)
$$

where $\theta_{1}(t)=\sum_{k=1}^{\infty} \exp \left(-8 \pi^{2} k^{2} t\right)$, a quantity independent of $\ell$. In particular the degeneration of the spectrum of a collar is independent of the boundary values. By a further analysis we find that (see Sects. 4.2 and 4.3)

$$
\theta_{*}(t)=e^{-t / 4}\left(\frac{\log l / \ell}{\sqrt{\pi t}}+O(1)\right) \text { for } t \geqq 1 \quad \text { as } \quad \ell \rightarrow 0 .
$$

We would like to compare this case to that of a flat Euclidean torus of meridian $\varepsilon / 2$ and area 1 . Let the spectral counting function be $N_{\varepsilon}(x)=\#\left\{\lambda_{n}\right.$ for $\varepsilon$-torus, $\left.\lambda_{n} \leqq x\right\}$ and $N_{\ell}(x)$ the spectral counting function for a compact hyperbolic surface $R_{\ell}$ with one short geodesic of length $\ell$. Then for fixed $x, N_{\varepsilon}(x) /$ diam ( $\varepsilon$-torus) $\rightarrow \frac{2}{\pi} \sqrt{x}$ and $N_{\ell}(x) / \operatorname{diam}\left(R_{\ell}\right) \rightarrow \frac{2}{\pi} \sqrt{x-\frac{1}{4}}$. In both cases the rescaled counting function converges to a universal expression, the counting function of a 1-dimensional problem. Our analysis confirms one's intuition. The clustering of the spectrum for a collar and a torus occurs only for the rotationally invariant functions, a 1-dimensional eigenvalue problem. Obviously the intuition is for low frequency waves traveling across a long cylinder. The comparison breaks down for the waves which oscillate along the meridians. In the Euclidean case the corresponding eigenvalues tend to infinity, while in the hyperbolic case the lowest frequency for a particular rotational mode actually remains bounded. The apparent similarity of the spectral divergences is more elementary than that predicted by Polyakov's vanishing of the conformal anomaly, [4]. We are merely considering the Laplacian on functions. Furthermore the divergence is the same whether the collar separates (the tachyon) or does not (an internal line of the diagram).

Our detailed analysis is guided by two conjectures. Let $\mathscr{Z}(s, \ell)$ $=\sum_{k=0}^{\infty}\left(1-e^{-(s+k) \ell}\right)^{2}$ be the contribution to the Selberg zeta product of a single geodesic of length $\ell$.

Conjecture 1. There exists a constant $c$, such that given a compact set $K \subset\left\{\operatorname{Re} s>\frac{1}{2}\right\}$, there exist positive constants $\alpha$ and $\beta$, depending only on $K$ and the genus, with

$$
\alpha \leqq|Z(s)| \prod_{\ell_{j}<c} \mathscr{Z}\left(s, \ell_{j}\right) \prod_{0<\lambda_{n}<\frac{1}{4}}\left(s^{2}-s+\lambda_{n}\right) \mid \leqq \beta
$$

for $s \in K$.

We prove that the conjecture is true for a neighborhood, depending only on the genus, of $s=1$ and for $\operatorname{Re} s>1$. For $\operatorname{Re} s<\frac{1}{2}$ the symmetry of $Z$ and the behavior of $\mathscr{Z}$ force the quotient to be unbounded as the $\ell_{j} \rightarrow 0$. 
If $R_{\tau} \rightarrow R_{o}\left(R_{\tau}\right.$ smooth, $R_{o}$ a surface with nodes $)$ we write $R_{o}-\{$ nodes $=S_{1} \cup \ldots \cup S_{q}$, a union of components and define $Z\left(s, R_{o}\right)=\prod_{k=1}^{q} Z\left(s, S_{k}\right)$. The Selberg zeta function $Z\left(s, S_{k}\right)$ is defined by the infinite product over geodesic lengths

$$
Z\left(s, S_{k}\right)=\prod_{\gamma} \prod_{k=0}^{\infty}\left(1-e^{-(s+k) \ell(\gamma)}\right)^{2} \quad \text { for } \quad \operatorname{Re} s>1
$$

and has a meromorphic continuation to the entire $s$-plane, $[38,40]$.

Conjecture 2. For $\operatorname{Re} s>\frac{1}{2}$

$$
Z\left(s, R_{\tau}\right) / \prod_{\ell_{j}<c} \mathscr{Z}\left(s, \ell_{j}\right) \rightarrow Z\left(s, R_{o}\right)
$$

We establish the second conjecture also for a neighborhood, depending only on the genus, of $s=1$, and for $\operatorname{Re} s>1$. Again the symmetry of $Z$ shows that the statement is false for $\operatorname{Re} s<\frac{1}{2}$.

Both conjectures imply that the order of the zero of the limit of $Z^{\prime}\left(s, R_{\tau}\right) / \prod_{\ell_{j}<c} \mathscr{Z}\left(s, \ell_{j}\right)$ at $s=1$ is the number of components of $R_{o}-\{$ nodes $\}$ (recall that $Z(\mathrm{~s})$ has a simple zero at $s=1)$. This is no surprise, the values $Z(1)$ and $Z^{\prime}(1)$ are finite multiplicative in the eigenvalues and $q-1$ eigenvalues are approaching zero.

\section{A Discussion of the Results}

First we should make two comments on our notation. In passing from one theorem to the next we may not choose new notation for our constants. Also we shall write all eigenvalues as non-negative and the first is always $\kappa_{1}$ (be it 0 or not).

Chapter 2. The fundamental example of degeneration is the family $\{z w$ $=t|| z|| w,|| t \mid,<1\}$ considered as a fibre space over the disc $\{|t|<1\}$. The $t \neq 0$ fibre is $\{|t|<|z|<1\}$ with hyperbolic metric $\left(\frac{\pi}{\log |t|} \csc \left(\pi \frac{\log |z|}{\log |t|}\right)\left|\frac{d z}{z}\right|\right)^{2}$. The $t=0$ fibre is (we remove the origin by convention) $\{|z|<1\} \cup\{|w|<1\}$ with metric $\left(\frac{|d \zeta|}{|\zeta| \log |\zeta|}\right)^{2}$ on each piece. In order to have an idea of Teichmüller space and degeneration, by analogy with this example, we describe the tangent and cotangent spaces of this family and also the Weil-Petersson metric. In the proof of Lemma 2.1 we show how to estimate the degeneration of a hyperbolic metric on a compact surface in terms of this example. The method is an improved version of Masur's argument, [22].

There are several definitions of a collar which are essentially equivalent. The fundamental point is that there exists an absolute constant $c_{*}$ (the collar constant) such that if a geodesic $\gamma$ has length $\ell(\gamma) \leqq c_{*}$ then the geometry of a tubular neighborhood $C(\ell(\gamma))$ of $\gamma$ of width $2 \log 2 / \ell$ depends only on $\ell(\gamma)$, i. e. is otherwise independent of the surface $\gamma$ lies on. To see that the geometry of the collar complement is uniformly bounded we introduce a construction. Given a surface $R$ remove the collars about the short geodesics and double the remaining surface to 
obtain $\tilde{R}$, the fattened double. $\widetilde{R}$ has a Finsler metric (a continuous line element) and the important point is that the moduli space $\{\widetilde{R}-$ Finsler metrics $\} /$ Diff is compact (Lemmas 2.2 and 2.3).

Chapter 3. The final theorem (Theorem 3.6) summarizes the discussion. Given $R$ with $m$ short geodesics, associated collars $C_{1}, \ldots, C_{m}$ and such that $\widetilde{R}$ has $q$ components we wish to estimate $\theta_{*}(t)$. As further notation we write $\theta(t, P, n)$ for the heat trace of the eigenvalue problem $P$ with the first $n$ eigenvalues deleted. Examples for $P$ are $D C$ the Dirichlet problem for a collar $C$ and $E \widetilde{R}, O \widetilde{R}$, the even and odd functions on $\widetilde{R}\left(\tilde{R}\right.$ has the doubling reflection). The theta function $\theta_{1}(t)$ was defined above. By eigenvalue comparison arguments we find that there exists a $\varrho>0$ such that

$$
\begin{aligned}
\theta(t, O \tilde{R}) & +\sum_{j=1}^{m} \theta\left(t, D C_{j}\right)-(2 m+q) e^{-\varrho t} \leqq \theta(t, R, 2 m+q) \\
& \leqq \theta(t, E \tilde{R}, q)+\sum_{j=1}^{m} \theta\left(t, D C_{j}\right)+4 m \theta_{1}(t)
\end{aligned}
$$

and the first eigenvalue for each term is bounded below by $\varrho$.

Chapter 4. By an elementary version of the Selberg trace formula we find

$$
\theta(t, D C) \leqq k(t) \text { Area }(C)+\frac{e^{-t / 4}}{\sqrt{\pi t}}\left(\log \frac{1}{\ell}+c\right),
$$

where again $k(t)$ is the elementary heat kernel evaluated at the source. Thus $\frac{e^{-t / 4}}{\sqrt{\pi t}} \log \frac{1}{\ell}$ is an upper bound for the divergence in $\ell$ of $\theta(t, D C)$. For a lower bound we consider only the rotationally invariant functions on the collar. The corresponding trace already contributes the same $\ell$-divergence; the remaining spectrum is bounded in $\ell$. Theorem 4.4 is our final bound for the trace of the heat kernel. In Sect. 4.5 we compare our estimate for the eigenvalues: $\kappa_{n}$ is of order $\left(\frac{1}{4}+\left(\frac{\pi n}{\operatorname{diam}}\right)^{2}\right)$ with the estimate of S.Y. Cheng: $\kappa_{n+1} \leqq\left(\frac{1}{4}+\left(\frac{4 \pi n}{\text { diam }}\right)^{2}\right)$, both for a compact surface, [8].

Chapter 5. We combine our considerations and McKean's formula to estimate $Z(s)$. By a standard argument $\mathscr{Z}(s, \ell) \approx \exp \left(-\pi^{2} / 3 \ell\right) \ell^{1-2 s}$ for $\operatorname{Re} s$ positive. We consider a surface $R$ with short geodesics of lengths $\left\{\ell_{j}\right\}_{j=1}^{m}$. There are three estimates with the constants depending only on the genus (except as noted).

1. There exists a neighborhood $N$ of $s=1$ such that given a compact set $K \subset N \cup\{\operatorname{Re} s>1\}$ there exists a positive constant $A(K)$ such that for $s \in K$

$$
A(K)^{-1} \leqq|Z(s)| \prod_{j=1}^{m} \mathscr{Z}\left(s, \ell_{j}\right) \prod_{0 \leqq \lambda_{n}<\frac{1}{4}}\left(s^{2}-s+\lambda_{n}\right) \mid \leqq A(K) .
$$

2. There exists a positive constant $B$ such that

$$
B^{-1} \leqq Z^{\prime}(1) / \prod_{j=1}^{m} \exp \left(-\pi^{2} / 3 \ell_{j}\right) \ell_{j}^{-1} \prod_{0<\lambda_{n}<\frac{1}{4}} \lambda_{n} \leqq B .
$$


3. For $N$ as defined above

$$
Z\left(s, R_{\tau}\right) / \prod_{j=1}^{m} \mathscr{Z}\left(s, \ell_{j}\right) \rightarrow Z\left(s, R_{o}\right)
$$

for $R_{\tau} \rightarrow R_{o}$, uniformly on compact subsets of $N \cup\{\operatorname{Re} s>1\}$. Results 1 and 2 are our estimate for the Polyakov integrand.

Chapter 6. We combine our results to obtain an estimate for the Polyakov string. In Sect. 6,2 we compare our estimate, given in terms of geodesic lengths, to that of Belavin-Knizhnik, given in terms of holomorphic coordinates [5]. Section 6.3 is a review of the Fenchel-Nielsen geometry of a neighborhood of the compactification divisor. Finally in Sect. 6.4 we combine our methods to show that the bosonic integral is divergent. To this end let $\mathscr{D} \subset \overline{\mathscr{M}}_{g}$ be the divisor of Riemann surfaces with nodes, $\tau$ a holomorphic parameter for the transverse direction to $\mathscr{D}$ and $(\ell, \theta)$ the Fenchel-Nielsen coordinates for the transverse. The variable $\tau$ parametrizes a family $R_{\tau}$ of Riemann surfaces. Recall that the pair $(\ell, \theta)$ is determined from $\tau$ as follows: first solve the constant curvature equation, $-\Delta_{\varrho} \log \varrho=-1$ on $R_{\tau}$, then find the length $\ell$ and twist $\theta$ for the geodesics in particular free homotopy classes. By using the maximum principle we are able to estimate solutions; in Sect. 2.5 we find the comparison $\ell \sim 2 \pi^{2} / \log 1 /|\tau|$ between the length and holomorphic parameters. Given that the Laplacian occurs in the constant curvature equation it is not hard to imagine that the lower order terms for the above expansion could involve the small eigenvalues. We do not know the answer to this question and are thus unable to find the lower order terms for the asymptotics of the Polyakov integrand. A discussion of these terms is contained in [39].

\section{Collars and the Degeneration Paradigm}

The fundamental observation on the degeneration of 2-dimensional conformal structures and similarly hyperbolic metrics is that the deformation localizes into collar neighborhoods about the short geodesics. This is very nice since the geometry of a collar is essentially independent of the surface; it is completely determined by the length of the core geodesic. Ideally, the asymptotic behavior on the moduli space $\mathscr{M}_{g}$ of an analytic invariant of a Riemann surface would be given by computing the invariant for the collars. The purpose of this chapter is to give an exposition of the techniques for and examples of rigorous arguments following this intuition.

In Sect. 2.2 we discuss the paradigm for the general case, the degeneration of an annulus. The special feature of the annulus is that everything can be worked out explicitly. Indeed in Sect. 2.3 we give the correspondence between tangent (respectively cotangent) vectors to Teichmüller space and Beltrami (respectively quadratic) differentials on the surface. We also calculate the Weil-Petersson metric and find its asymptotic behavior. Section 2.4 is a review of the basic facts about collars. In Sect. 2.5 we show how the case of an annulus is used to construct deformations of a compact surface with nodes. An estimate for the comparison between the hyperbolic metrics of a family of annuli and a family of compact surfaces is given in Lemma 2.1. Finally, a method for bounding the geometry in the complement of the collars is given in Sects. 2.6 and 2.7. 
2.2 We start with a fibre space $\mathscr{F}$ over the disc $D=\{|t|<1\} . \mathscr{F}$ is the analog of the universal curve, (universal curve $=$ the fibre space with $\varrho$-fibre, $\varrho \in \mathscr{M}_{g}$, the Riemann surface represented by $\varrho$ (modulo symmetries)) and $D$ is the analog of the moduli space $\overline{\mathscr{M}}_{g}$. The locus $\mathscr{F}=\{z w=t|| z|| w,|| t \mid,<1\}$, the degeneration fixture, is a complex manifold fibering over $D$. To have an idea of the geometry consider the real points of $\mathscr{F}$. The fibres are hyperbolas moving to the coordinate axes and passing through. An alternate description is given by domains in $\mathbb{C}$ and hyperbolic geometry. The $t \neq 0$ fibres are the annuli $\{|t|<|z|<1\}$ with complete hyperbolic metrics $d s^{2}=\left(\frac{\pi}{\log |t|} \csc \left(\pi \frac{\log |z|}{\log |t|}\right)\left|\frac{d z}{z}\right|\right)^{2}$. The $t=0$ fibre is singular and is the union of the discs $\{|z|<1\}$ and $\{|w|<1\}$ joined at the origin. To obtain a hyperbolic metric remove the origin, then each punctured disc has a complete hyperbolic metric, i.e. on $\{0<|z|<1\} d s^{2}=\left(\frac{|d z|}{|z| \log |z|}\right)^{2}$. The first observation is that $d s_{t}^{2} \rightarrow d s_{0}^{2}$ real analytically in $(\log 1 /|t|)^{-1}$ uniformly for $|z|,|w|>\varepsilon$. This is essentially optimal since the metrics evaluated at $|z|=|t|^{1 / 2}$ are not even asymptotic. In particular it is false that the metrics converge uniformly to the limiting metric. A second observation concerns the length of the closed geodesic in the $t$-fibre. The geodesic is $|z|=|t|^{1 / 2}$ and has length $\ell=2 \pi^{2} /(\log 1 /|t|)$.

2.3. An example of obtaining the limiting behavior of a quantity on $\mathscr{M}_{g}$ from the annulus example is Masur's calculation of the Weil-Petersson metric. Recall that the tangent (respectively cotangent) space of Teichmüller space is isomorphic to a quotient of the space of Beltrami differentials (respectively the space of holomorphic quadratic differentials), [35]. To make the analogy of $\mathscr{F}$ over $\mathscr{D}$ to the genus $g$ universal curve $\mathscr{C}_{g}$ over $\mathscr{M}_{g}$ we must find the Beltrami differential on the Riemann surface $\{|t|<|z|<1\}$ that corresponds to the tangent vector $\frac{\partial}{\partial t}$ on the moduli space $D$ as well as the quadratic differential that corresponds to $d t$.

We consider the tangent vector first. The quasiconformal map $f(z)=z|z|^{s}, s$ real, maps the annulus $\{|t|<|z|<1\}$ to $\left\{|t|^{s+1}<|z|<1\right\}$. For $s=0,\left(f_{s}\right)_{\bar{z}}=z / 2 \bar{z}$ is the Beltrami differential and furthermore $\frac{d|t|^{s+1}}{d s}=t \log |t|$. Thus $\left(f_{t}\right)_{\bar{z}}=s_{t}\left(f_{s}\right)_{\bar{z}}$ $=\frac{1}{2 t \log |t|} \frac{z}{\bar{z}}$ is the Beltrami differential on $\{|t|<|z|<1\}$ representing $\frac{\partial}{\partial t}$ at $t \in D$. The next item is to find the quadratic differential representing $d t$. The Teichmüller theory of the annulus tells us that the differential is $c\left(\frac{d z}{z}\right)^{2}$ for $c$ real and that the pairing is by integration [35]. Thus $1=\left(\frac{\partial}{\partial t}, d t\right)=\frac{c}{2} \int\left(\frac{1}{t \log |t|} \frac{z}{\bar{z}}\right)\left(\frac{1}{z^{2}}\right) d E$, where $d E$ is the Euclidean form and the domain is $\{|t|<|z|<1\}$. Evaluating the integral we find that $d t$ is represented by $\varphi=\frac{-t}{\pi}\left(\frac{d z}{z}\right)^{2}$.

The definition of the Weil-Petersson pairing involves projection onto the harmonic Beltrami differentials. This step may be avoided by computing the dual metric for quadratic differentials. By definition $\langle d t, d t\rangle=\int|\varphi|^{2} \lambda^{-2} d E$, where 
$\lambda^{2}=\left(\frac{\pi}{|z| \log |t|} \csc \left(\pi \frac{\log |z|}{\log |t|}\right)\right)^{2}$ is the density for the hyperbolic metric. In brief the result of integrating over the annulus $\{|t|<|z|<1\}$ is $\langle d t, d t\rangle=|t|^{2}\left(\frac{1}{\pi} \log 1 /|t|\right)^{3}$, the Hermitian (complex form) pairing on the holomorphic cotangent space. The Riemannian (real form) W-P metric is then $d s_{W P}^{2}=\frac{2 \pi^{3}|d t|^{2}}{|t|^{2}(\log 1 /|t|)^{3}}$. As a check we point out that this agrees exactly with our formula $d \tau \wedge d \ell$ for the W-P volume form [33]. In particular if $(\tau, \ell)$ are Fenchel-Nielsen coordinates (see Sect. 6.4) then from the paradigm the change of variables is $\ell=2 \pi^{2} /(\log 1 /|t|)$ and $2 \pi \tau / \ell=\arg t$ $=\operatorname{Im} \log t$.

2.4. For the above model the collar is the subannulus $\left\{|t| e^{2 \pi} \leqq|z| \leqq e^{-2 \pi}\right\}$ of $\{|t|<|z|<1\}$. The main result is that there exists a positive constant $c_{*}$ such that if the length $\ell$ of a geodesic $\gamma$ on a surface $R$ satisfies $\ell \leqq c_{*}$, then the standard collar embeds isometrically about $\gamma,[23,27]$. With the degeneration fixture we can check that as $\ell \rightarrow 0$ a collar converges to the union of a pair of disjoint (area 1) neighborhoods of cusps.

We review the basic properties of the collar. A standard model for the collar is described as a quotient of the upper half plane $H$. The deck transformation $z \rightarrow e^{\ell} z$ generates a cyclic group $\Gamma_{\ell}$ with $H / \Gamma_{\ell}$ a hyperbolic annulus with core geodesic of length $\ell$. By definition the standard collar is the $\Gamma_{\ell}$ quotient of the wedge $\{\ell<\arg z$ $<\pi-\ell\}$. Let $R$ be a compact surface with hyperbolic metric. Consider $\gamma$ a geodesic on $R$ of length $\ell \leqq 2 c_{*}$, where $c_{*}$ is the collar constant (we shall make a finite number of adjustments to its definition in the following chapters).

1) The standard collar is isometric to a neighborhood of $\gamma$, the collar about $\gamma$.

2) Collars about distinct geodesics are disjoint and there are at most $3 g-3$.

3) The injectivity radius of $R$-\{collars $\}$ is bounded from below.

4) The width of a collar is $\sim 2 \log \frac{2}{\ell}$, its area $\approx 2$ and each boundary is of length $\approx 1$.

Geodesics of length at most $c_{*}$ will be called short geodescis.

2.5. We would like to explain how the degeneration fixture is the model for the general case. To do this it is easiest to start at infinity, the compactification divisor $\mathscr{D}$ of $\overline{\mathscr{M}}_{g}$, and then move back into $\mathscr{M}_{g}$. A point of $\mathscr{D}$ represents a Riemann surface $R$ with nodes. By definition a neighborhood of a point $p$ of $R$ is complex isomorphic to either $\{|z|<\varepsilon\}$ or $\{z w=0|| z|| w \mid,<\varepsilon\}$. Obviously in the latter case $p$ is a node. To parametrize opening the node first identify $U=\{z w=0|| z|| w \mid,<\varepsilon\}$ with the 0 -fibre of a family $\{z w=t|| z|| w,|<\varepsilon| t \mid,<\varepsilon\}$. A deformation of $R \in \bar{M}_{g}$ which opens the node is given by varying the parameter $t$. Specifically for $t \neq 0$ the fibre of the family is a single annulus obtained by identifying $\{|t| / \varepsilon<|z|<\varepsilon\}$ with $\{|t| / \varepsilon<|w|<\varepsilon\}$ by the map $w=t / z$; the boundaries $\{|z|=\varepsilon\},\{|w|=\varepsilon\}$ of the respective $z$ and $w$ annuli are independent of $t$ and are attached to $R-U$ in the same way as for $U$ itself.

In order to elaborate on the above idea we shall recall Masur's description of a finite manifold cover of a neighborhood of $R_{0} \in \mathscr{D} \subset \bar{M}_{g},[22 ; 37$, pp. 37, 50]. 
Denote by $p_{1}, \ldots, p_{m}$ the nodes of $R_{0}$; for the node $p_{i}$ the punctures $a_{i}$ and $b_{i}$ of $R_{o}-\left\{p_{1}, \ldots, p_{m}\right\}$ are paired. Let $U=\{|z|<1\} ; U_{i}^{1}, U_{i}^{2}, i=1, \ldots, m$ be disjoint neighborhoods of the punctures $a_{i}$ and $b_{i}$. Now choose local coordinates $z_{i}: U_{i}^{1} \rightarrow U$ and $w_{i}: U_{i}^{2} \rightarrow U$ with $z_{i}\left(a_{i}\right)=0$ and $w_{i}\left(b_{i}\right)=0$. Fix an open set $\mathscr{V} \subset R_{o}$ disjoint from the sets $U_{i}^{1}, U_{i}^{2}$. Beltrami differentials $\mu_{j}$ with support in $\mathscr{V}$ are chosen to span the tangent space of the (product) Teichmüller space of $R_{o}-\left\{p_{1}, \ldots, p_{m}\right\}$ (the dimension is $3 g-3-m)$. Given $s=\left(s_{1}, \ldots, s_{3 g-3-m}\right) \in \mathbb{C}^{3 g-3-m}$ for a neighborhood of the origin the sum $\mu(s)=\sum_{j} s_{j} \mu_{j}$ satisfies $\|\mu(s)\|_{\infty}<1$, and thus a solution $\omega^{\mu(s)}$ of the Beltrami equation exists. The surface $\omega^{\mu(s)}\left(R_{o}\right)=R_{s}$ is a quasiconformal deformation of $R_{o}$.

Now to parametrize the opening of the nodes. The map $\omega^{\mu(s)}$ is conformal on $U_{i}^{1}, U_{i}^{2}$ and therefore $z_{i}$ and $w_{i}$ also serve as local coordinates for $\omega^{\mu(s)}\left(U_{i}^{1}\right)$, $\omega^{\mu(s)}\left(U_{i}^{2}\right) \subset \omega^{\mu(s)}\left(R_{o}\right)$. Given $\sigma=\left(\sigma_{1}, \ldots, \sigma_{m}\right) \in \mathbb{C}^{m},\left|\sigma_{i}\right|<1$ we construct the surface $R_{\sigma, s}$ as follows. Remove the discs $\left\{0<\left|z_{i}\right| \leqq\left|\sigma_{i}\right|\right\}$ and $\left\{0<\left|w_{i}\right| \leqq\left|\sigma_{i}\right|\right\}$ from $R_{s}$. Attach $\left\{\left|\sigma_{i}\right|<\left|z_{i}\right|<1\right\}$ to $\left\{\left|\sigma_{i}\right|<\left|w_{i}\right|<1\right\}$ by identifying $z_{i}$ to $\sigma_{i} / w_{i}$. The construction is complete; the tuple $(\sigma, s)$ parametrizing $R_{\sigma, s}$ provides a local complex coordinate chart $\mathscr{C}$.

In the following we shall refine Masur's discussion to establish an estimate for the length $\ell_{i}$ of the geodesic, homotopic to the core of the $\sigma_{i}$-annulus. From the degeneration fixture we expect an answer $\ell_{i} \sim 2 \pi^{2} /\left(\log 1 /\left|\sigma_{i}\right|\right)$ or equivalently $\ell_{i} \log 1 /\left|\sigma_{i}\right| \sim 2 \pi^{2}$.

Lemma 2.1. Given $\varepsilon>0$ there exists a neighborhood $\mathscr{C}_{o} \subset \mathscr{C}$ of $R_{o}$ such that for $(\sigma, s) \in \mathscr{C}_{o}$,

$$
\left|2 \pi^{2}-\ell_{i} \log 1 /\right| \sigma_{i}||<\varepsilon \quad i=1, \ldots, m
$$

and on each $\sigma_{i}$-annulus the $R_{\sigma, s}$ hyperbolic metric is asymptotic to the degeneration fixture.

Before the proof we follow Masur and review an argument of Ahlfors, [3]. Let $\lambda$ and $\lambda_{*}$ be metrics of constant curvature -1 for a non-compact surface $S$ (neither need be complete); the curvature equation is $\Delta \log \lambda=\lambda^{2}, \Delta \log \lambda_{*}=\lambda_{*}^{2}$. Thus $\Delta$ $\log \left(\lambda / \lambda_{*}\right)=\lambda^{2}-\lambda_{*}^{2}$ and the maximum principle provides that $\log \left(\lambda / \lambda_{*}\right)$ cannot have a positive local maximum. Thus on a compact subdomain $K \subset S, \lambda / \lambda_{*}$ is bounded by the greater of 1 and the maximum of the ratio on the boundary. This is Ahlfors' observation.

Proof of Lemma 2.1. The key point is to make a judicious choice of the charts $z_{i}$ and $w_{i}$ in Masur's discussion. The argument is illustrated by the case $z_{1}, w_{1}$; to simplify notation we omit the index. Since $a$ and $b$ are cusps of $R_{o}-\{$ nodes $\}$ we can choose charts $z$ and $w$ such that the hyperbolic metric of $R_{o^{-}}$\{nodes $\}$restricts on $U^{1}$ and $U^{2}$ to $\Lambda_{o}=\left(\frac{|d \zeta|}{|\zeta| \log |\zeta|}\right)^{2}$ on $\left\{0<|\zeta|<c_{o}\right\}$ for $z=\zeta$ and for $w=\zeta\left(c_{o}<1\right.$ is the collar constant for cusps). For the construction of the coordinate $\sigma$ we shall use the subfamily $\left\{z w=\sigma|| z|| w,\left|<c_{o},\right| \sigma \mid<c_{o}^{2}\right\}$ of the degeneration fixture with the restriction hyperbolic metric on each fibre, denoted by $\Lambda_{\sigma}$.

The subfamily has a second hyperbolic metric. In Masur's discussion the subfamily is realized as a subdomain of the surface $R_{\sigma, s}$. The hyperbolic metric of 
$R_{\sigma, s^{-}}\{$nodes $\}$restricts to the subfamily; denote the restriction by $\lambda_{\sigma, s}$. Our first estimate is a comparison of $\Lambda_{o}$ and $\lambda_{\sigma, s}$. By definition of the charts $z$ and $w$ we have that $\lambda_{o, o}=\Lambda_{o}$. By a result of Bers [6], away from the nodes, $\lambda_{\sigma, s}$ varies continuously with $(\sigma, s)$. Thus given $\varepsilon>0$ there exists a neighborhood $\mathscr{C}_{o}$ of $R_{o}$ such that $(1-\varepsilon) \lambda_{\sigma, s}$ $\leqq \Lambda_{o} \leqq(1+\varepsilon) \lambda_{\sigma, s}$ for $|z|=c_{o} / 2 ;|w|=c_{o} / 2$ and $(\sigma, s) \in \mathscr{C}_{o}$. This is the first estimate.

The second estimate is for the deformation fixture $\{z w=\sigma|| z|| w,|,| \sigma \mid<1\}$. As discussed in Sect. 2.2 the hyperbolic metric $\Lambda_{\sigma}$ of the $\sigma$-annulus converges to the metric of the 0 -fibre for $|z|$ fixed and for $|w|$ fixed. Thus there exists a $\delta>0$ such that $(1-\varepsilon) \Lambda_{o} \leqq \Lambda_{\sigma} \leqq(1+\varepsilon) \Lambda_{o}$ for $|\sigma|<\delta$ and $|z|=c_{o} / 2,|w|=c_{o} / 2$. This is the second estimate.

Now on the compact subannulus $\left\{2|\sigma| / c_{o} \leqq|z| \leqq c_{o} / 2\right\}, \sigma \neq 0$, we apply the Ahlfors argument to $\lambda_{\sigma, s} / \Lambda_{\sigma}$ and to $\Lambda_{\sigma} / \lambda_{\sigma, s}$. Denoting by $M$ and $m$ the maximum and minimum of $\Lambda / \lambda$ on the boundary of the annulus, then $\min (1, m) \leqq \Lambda / \lambda$ $\leqq \max (1, M)$. With this estimate the problem is reduced to considering the behavior on the boundaries of the subannuli. Combining the first and second estimates (assume $|\sigma|<\delta$ ) we find

$$
(1-\varepsilon)^{2} \leqq(1-\varepsilon) \Lambda_{\sigma} / \Lambda_{o} \leqq \Lambda_{\sigma} / \lambda_{\sigma, s} \leqq(1+\varepsilon) \Lambda_{\sigma} / \Lambda_{o} \leqq(1+\varepsilon)^{2}
$$

In summary we have that $(1-\varepsilon)^{2} \leqq \Lambda_{\sigma} / \lambda_{\sigma, s} \leqq(1+\varepsilon)^{2}$ on the entire subannulus. Recall by the Bers result $\varepsilon \rightarrow 0$ as $(\sigma, s) \rightarrow(0,0)$ and thus the $R_{\sigma, s}$ metric is asymptotic to the deformation fixture.

Finally, as an application we estimate the lengths of the core geodesics. Recall that the $\Lambda_{\sigma}$ geodesic has length $L=2 \pi^{2} / \log (1 /|\sigma|)$ and the $\lambda_{\sigma, s}$ geodesic has length $\ell$ bounded as $(1-\varepsilon)^{2} \leqq L / \ell \leqq(1+\varepsilon)^{2}$, the desired conclusion.

2.6. We have seen that the degeneration of a hyperbolic metric is associated to the formation of wide collars about short geodesics. An important point is the behavior of the metric in the complement of the collars. It is known that in the collar complement the geometry is uniformly bounded. We shall establish this result by an explict construction. The idea is to start with a surface $R$, remove the collars about the short geodesics and glue the resulting boundaries in pairs to construct an associated surface $\hat{R}$. In fact the set of associated surfaces $\left\{\hat{R} \mid R \in \overline{\mathscr{M}}_{g}\right\}$ form a compact subset of $\mathscr{M}_{g}$. The last observation will be essential for estimating the spectrum.

We start with the construction. The basic idea is illustrated for the case of an annulus $A$. If the covering transformation is $z \rightarrow e^{\ell} z$, then a fundamental domain is $\left\{1<|z|<e^{\ell}\right\}$ and the standard collar $C$ lifts to the wedge $\{\ell<\arg z<\pi-\ell\}$. Choose a smooth monotone function $\varphi$ such that $\varphi(\ell)=2 \ell$ for $\ell \leqq c_{*}$ and $\varphi(\ell)=\pi / 2$ for $\ell \geqq 2 c_{*}$. We define the standard subcollar $S C$ as the quotient of $\{\varphi(\ell)<\arg z<\pi-\varphi(\ell)\}$. The plan is to consider $C-S C$ and identify the inner boundaries. Letting $w$ be the coordinate of the new space then

$$
\begin{aligned}
& w=z \exp \left(i\left(\frac{\pi}{2}-\varphi(\ell)\right)\right) \quad \text { for } \quad \ell<\arg z<\varphi(\ell) \text { and } \\
& w=z \exp \left(-i\left(\frac{\pi}{2}-\varphi(\ell)\right)\right) \quad \text { for } \quad \pi-\varphi(\ell)<\arg z<\pi-\ell ;
\end{aligned}
$$


$w$ varies in the wedge $\ell-\varphi(\ell)<\arg w-\frac{\pi}{2}<\varphi(\ell)-\ell$. The deck transformation $z \rightarrow e^{\ell} z$ is compatible with the above glueing and thus we can form the quotient of the $w$-wedge to obtain the associated surface (by definition) $\hat{C}$. The restriction of the hyperbolic metric $d s^{2}$ of $A$ to $C-S C$ defines a Finsler metric (a continuous length element), the associated metric, $\left(\hat{d} s^{2}\right)$ on $\hat{C}$. It is the geometry of $\hat{C}$ that we wish to consider. By definition $\left(\hat{d} s^{2}\right)$ in $w=r e^{i \theta}$ polar coordinates is

$$
\left(\hat{d} s^{2}\right)=\frac{d r^{2}+r^{2} d \theta^{2}}{r^{2} \sin ^{2}\left(\varphi(\ell)-\left|\frac{\pi}{2}-\theta\right|\right)}
$$

The width of $\hat{C}$ is bounded below independent of $\ell$; the width is $2 \int_{\ell}^{2 \ell} \csc \theta d \theta \approx 2 \log 2$ for small $\ell$. The second observation is that the closed curve on $\hat{C}$ covered by $\arg w=\frac{\pi}{2}$ is the shortest in its free homotopy class; its length is $\ell \csc 2 \ell \approx \frac{1}{2}$ for small $\ell$.

An important observation concerns the curvature of $\left(\hat{d} s^{2}\right)$. For $\arg w \neq \frac{\pi}{2}$ the metric is smooth and certainly has curvature -1 . For $\arg w=\frac{\pi}{2}$ the metric coincides with the comparison metric $d s_{c}^{2}=\frac{d r^{2}+r^{2} d \theta^{2}}{r^{2} \sin ^{2}\left(\theta-\frac{\pi}{2}+\varphi(\ell)\right)}$ and in general $\left(d \hat{s}^{2}\right) \geqq d s_{c}^{2}$. The comparison metric has curvature -1. In brief $\left(\hat{d} s^{2}\right)$ has a supporting metric with curvature -1 . This is precisely the hypothesis of the Ahlfors-Schwarz lemma, [3].

We will now check that the construction of $\hat{C}$ can also be carried out for the limit case $\ell=0$. Recall from Sect. 2.2 that the 0 -fibre of $\mathscr{F}$ is the union of $\{0<|z|<1\}$ with metric $\left(\frac{|d z|}{|z| \log |z|}\right)^{2}$ and $\{0<|w|<1\}$ with metric $\left(\frac{|d w|}{|w| \log |w|}\right)^{2}$. It is easy to check that the limit of the collar is $C=\left\{0<|z|<e^{-2 \pi}\right\} \cup\left\{0<|w|<e^{-2 \pi}\right\}$; the limit of the subcollar is $S C=\left\{0<|z|<e^{-4 \pi}\right\} \cup\left\{0<|w|<e^{-4 \pi}\right\}$. We shall now describe the construction of $\hat{C}$ for this situation. Consider $C-S C$ and identify the inner boundaries $\left\{|z|=e^{-4 \pi}\right\},\left\{|w|=e^{-4 \pi}\right\}$ to obtain $\hat{C}=\left\{e^{-2 \pi}<|v|<e^{2 \pi}\right\}$ (i.e. $v=e^{4 \pi} z$, $\left.e^{-4 \pi}<|z|<e^{-2 \pi} ; \quad v=e^{-4 \pi} / w, e^{-4 \pi}<|w|<e^{-2 \pi}\right) \quad$ and $\quad\left(\hat{d} s^{2}\right)=\left(\frac{|d v|}{|v|(4 \pi-|\log | v||)}\right)^{2}$ with comparison metric $\hat{d} s_{c}^{2}=\left(\frac{|d v|}{|v|(4 \pi+\log |v|)}\right)^{2}$. The analogous statements for the width, area, and length of the core curve as well as the behavior of $d s_{c}^{2}$ are valid for this case also.

Now we shall explain how the case of an annulus is the model for the general case, a Riemann surface $R$ with nodes $\left\{p_{1}, \ldots, p_{m}\right\} \quad(m \geqq 0)$. Consider $R-\left\{p_{1}, \ldots, p_{m}\right\}$ with its hyperbolic metric. By the collar result a collar neighborhood $C$ of a short geodesic $\left(\ell \leqq c_{*}\right)$ or a collar neighborhood $C$ of a pair of 
punctures is isometric to the collar for an annulus. Perform the above construction replacing each collar $C$ of $R$ with the associated $\hat{C}$. This is the construction of $\hat{R}$, the associated surface. $\hat{R}$ is a compact surface without nodes, has a smooth conformal structure and so by the uniformisation theorem has a smooth hyperbolic metric $\Lambda(\hat{R})$.

Lemma 2.2. With the above notation,

1) There exists a positive constant $\alpha$ such that the shortest $\left(d \hat{s}^{2}\right)$ curve not homotopic to a point has length at least $\alpha$.

2) $\left(d \hat{s}^{2}\right) \leqq \Lambda(\hat{R})$.

3) The set $\left\{\hat{R} \mid R \in \overline{\mathscr{M}}_{g}\right\}$ of associated conformal structures (modulo diffeomorphisms) is compact.

4) The set of Finsler metrics (modulo diffeomorphism) $\left\{\hat{d s}^{2} \mid R \in \overline{\mathscr{M}}_{g}\right\}$ is compact.

Proof of 1). Let $\gamma$ be a closed curve on $\hat{R}$, not homotopic to a point. The first case to consider is that $\gamma$ does not intersect the surgery locus. For this case $\gamma$ can be considered as a curve (not homotopic to a non-nodal point) on the original surface $R$. There are two subcases: $\gamma$ is not homotopic to one of the short geodesics or a node, thus by definition $\ell(\gamma) \geqq c_{*}$ or $\gamma$ is homotopic to a short geodesic or a node but disjoint from the corresponding subcollar. We saw above for the basic construction that $\ell(\gamma)$ is bounded below in this situation.

The second case is that $\gamma$ does intersect the surgery locus. There are two subcases. First consider that $\gamma$ also contains a point outside the associated collar $\hat{C}$. In this situation $\ell(\gamma)$ is bounded by half the width of the collar which is itself bounded below. The only remaining situation is that $\gamma$ is contained in the collar $\hat{C}$. Since $\gamma$ is not homotopic to a non-nodal point it must represent a positive power of the core geodesic. We saw above that there is an absolute lower bound for the length of $\gamma$ in this circumstance. The proof is complete.

Proof of 2). By the construction of $\hat{R}$ the metric $\left(\hat{d s}^{2}\right)$ has a supporting metric of curvature -1 . The conclusion follows from the Ahlfors-Schwarz lemma, [3].

Proof of 3). By 1) and 2) for an associated surface $\hat{R}$ the length of the shortest $\Lambda(\hat{R})$ geodesic is at least $\alpha$. Recall that the set of surfaces with minimal geodesic of length at least $\alpha$ is a compact subset of $\mathscr{M}_{g}$, [25].

Proof of 4). This is a reformulation of the result of Bers [6, Sect. 3] that the hyperbolic metrics of a family of nodal surfaces varies continuously away from the nodes.

2.7. A basic technique for estimating the spectrum is to write the domain as a union and then estimate by the union of the spectra of the components. Accordingly we can try to think of the eigenfunctions of the entire domain as closely approximated by those of the components. The goal of Chaps. 3 and 4 is to show that the divergence of the spectrum of a compact surface is due to the divergence of the spectrum of the collars. A key step is to isolate and bound that part of the spectrum associated to the collar complement. The plan is to derive this result from bounds on the geometry of the complement. The argument is simplest for a variant of the construction from the previous section. 
Start with a surface $R$ (possibly with nodes), remove the standard subcollars about the short geodesics and double the resulting surface to obtain $\widetilde{R}$, the fattened double. In general $\widetilde{R}$ is not connected but the total genus is bounded since the Euler characteristic is $2 \chi(R)$.

The discussion of the previous section applies directly and we have that the doubled metric $\left(\widetilde{d} s^{2}\right)$ has a supporting metric of curvature -1 . $\widetilde{R}$ has a smooth conformal structure and by the uniformisation theorem a smooth hyperbolic metric $\Lambda(\widetilde{R})$. The proof of Lemma 2.2 may be applied verbatim.

Lemma 2.3. With the above notation,

1) There exists a positive constant $\alpha$ such that the shortest $\left(\tilde{d} s^{2}\right)$ curve not homotopic to a point has length at least $\alpha$.

2) $\left(\widetilde{d} s^{2}\right) \leqq \Lambda(\widetilde{R})$.

3) The set $\left\{\widetilde{R} \mid R \in \overline{\mathscr{M}}_{g}\right\}$ of fattened double conformal structures (modulo diffeomorphisms) is compact.

4) The set of Finsler metrics (modulo diffeomorphisms) $\left\{\tilde{d} s^{2} \mid R \in \overline{\mathscr{M}}_{g}\right\}$ is compact.

Finally to expedite certain technical arguments it may also be convenient to consider $R-S C=R-\bigcup_{j} S C_{j}$, one half of the double, as a Riemannian manifold with boundary. The geodesic curvature of a boundary component is an elementary function of the length of the removed core geodesic; the curvature is bounded in terms of the collar constant. An alternate formulation of Lemmas 2.2 and 2.3 is that the set of Riemannian manifolds with boundary $\left\{R-S C \mid R \in \overline{\mathscr{M}}_{g}\right\}$ (modulo diffeomorphisms) is compact relative to the $C^{\infty}$ topology on the space of Riemannian metrics.

\section{Bounding the Eigenvalue Spectrum of a Surface by the Spectrum of the Collars}

3.1 In Chap. 2 we saw that the degeneration of a hyperbolic metric is concentrated in the collars about short geodesics. Naively one might expect the same behavior for the spectrum. Any short geodesic divergence should come from the spectrum of a collar. Specifically, the divergence of the spectrum of the union of the collars might closely approximate that of the surface. For eigenvalues (positive by convention) above $\mathrm{a} \frac{1}{4}$ we shall establish a precise form of this statement with Theorem 3.6. For eigenvalues below $\frac{1}{4}$ this is actually false. In the latter case the behavior also depends on the location of the collars in the surface, not just their geometry (see Sect. 3.2). For example if exactly one geodesic is pinched $(\ell \rightarrow 0)$ then $\lambda_{1} \rightarrow 0$ if and only if the geodesic separates the surface.

Section 3.2 is a review of the behavior of the small eigenvalues $\left(<\frac{1}{4}\right)$. They will always be treated separately. If a surface $R$ is written as a disjoint union of $R$ \{subcollars\} and \{subcollars\} then the Neumann and Dirichlet spectra of the pieces provide bounds for the spectrum of $R$. This classical result is noted in Sect. 3.3. In the next section we present a variant of the classical estimate, the analogous result but with the small eigenvalues removed. Section 3.5 is devoted to estimating the difference between the trace of the Dirichlet and Neumann heat kernels for a subcollar. The main result, Theorem 3.5, shows that the difference of the traces is 
bounded, independent of the length of the core geodesic of the subcollar, by the heat trace of a 1-dimensional eigenvalue problem. We combine our considerations in the final section and show that the heat trace of $R$ is estimated by the Dirichlet heat trace of the collars and the heat trace of the collar complement.

3.2. We review the known results for the small eigenvalues. By convention we consider the eigenvalues as non-negative and with our (non-standard) indexing the first is $\kappa_{1}$, even if it is 0 . An analysis of the small eigenvalues is contained in $[7,11,30]$.

An $n$-disconnect $D$ is a collection of disjoint simple closed geodesics disconnecting the surface $R$ into $n$ pieces. The length $L(D)$ is simply the sum of the lengths of the geodescis. Let $L_{n}(R)=\min L(D)$, where the minimum is over all $n$-disconnects for a surface $R$.

Theorem 3.1 $[11,30]$. There exist positive constants $\alpha_{1}$ and $\alpha_{2}$ dependig only on the genus such that

$$
\alpha_{1} L_{n}(R) \leqq \kappa_{n} \leqq \alpha_{2} L_{n}(R)
$$

for $1 \leqq n \leqq 2 g-2$ and

$$
\alpha_{1} \leqq \kappa_{2 g-1} \leqq \alpha_{2}
$$

An estimate for the first eigenvalue above $\frac{1}{4}$ was given by Buser: $\kappa_{4 g-1} \geqq \frac{1}{4}$, [7] .

3.3 An argument by comparison theorems is formal; to emphasize the organization we wish to introduce certain abbreviations

$$
\kappa_{n}(P)=n^{\text {th }} \text { eigenvalue for the eigenvalue problem } P .
$$

Examples.

$\begin{array}{ll}R & \text { a compact surface, } \\ N S C & \text { s standard subcollar with Neumann boundary condition }\left(\frac{\partial u}{\partial n}=0\right),\end{array}$

$D S C$ a standard subcollar with Dirichlet boundary condition $(u=0)$,

$P_{1} \cup P_{2}$ the union of two eigenvalue problems, as a Hilbert space direct sum of operators.

Now consider a surface $R$ with short geodesics $\left(\ell \leqq c_{*}\right) \gamma_{j}$ and associated collars $S C_{j}, j=1, \ldots, m$. Recall the construction of the fattened double $\widetilde{R}$ (see Sect. 2.7) and let $q(\tilde{R})$ be its number of components. We shall abbreviate $R-S C$ for $R-\bigcup_{j=1}^{m} S C_{j}$ and $N(R-S C), D(R-S C)$ for the Neumann and Dirichlet problems of $\stackrel{i=1}{R-S C}$. We start with the classical estimate [31]

$$
\kappa_{n}\left(N(R-S C) \cup \bigcup_{j=1}^{m} N S C_{j}\right) \leqq \kappa_{n}(R) \leqq \kappa_{n}\left(D(R-S C) \cup \bigcup_{j=1}^{m} D S C_{j}\right) .
$$

By construction the double $\widetilde{R}$ has a continuous, piecewise smooth metric with doubling symmetry $r . L^{2}(\widetilde{R})$ is the direct sum of two subspaces: $E$, functions even with respect to $r$ and $O$, functions odd with respect to $r$. The smooth functions are dense in each subspace and the spectrum is defined as the successive infima of a 
quadratic form (the gradient integral). The spectral decomposition is the direct sum of the decompositions for the even and odd subspaces [41, esp. Sect. 5]. Denote by $E \widetilde{R}$ the spectral problem for the even subspace and by $O \widetilde{R}$ that of the odd subspace. It is immediate that $E \widetilde{R}$ is identifiable with $N(R-S C)$ and $O \widetilde{R}$ with $D(R-S C)$.

3.4. The goal of this section is to bound the trace $\theta_{e}(t, R)$ of the heat kernel of $R$ (with small eigenvalues excluded) below by that of $O \tilde{R} \bigcup_{j} D S C_{j}$ and above by $\tilde{E} R \bigcup_{j} N S C_{j}$. We must remove the small eigenvalues from the discussion; they are essential for estimating $Z^{\prime}(1)$ and (3.1) is totally inadequate. In particular $\kappa_{1}(N S C)=0$ and $\kappa_{2}(N S C) \rightarrow 0$ as $\ell \rightarrow 0$ as contrasted with $\kappa_{1}(D S C)>\frac{1}{4}$, [11]

To compare spectra we introduce the trace of the heat kernel. We shall consider the trace $\theta_{e}(t, P)$ of an eigenvalue problem $P$ with a certain number of small eigenvalues excluded

$$
\theta_{e}(t, P)=\sum_{n>e_{*}(P)}^{\infty} e^{-\kappa_{n} t}
$$

where $e_{*}(N S C)=2, \quad e_{*}(E \tilde{R})=q(\tilde{R}), \quad e_{*}(R)=2 m+q$ and $e_{*}\left(O \tilde{R} \cup \bigcup_{j=1}^{m} D S C_{j}\right)$ $=2 m+q(m$ is the number of short geodesics and $q$ the number of components of $\widetilde{R})$ and as always

$$
\theta(t, P)=\sum_{n=1}^{\infty} e^{-\kappa_{n} t}
$$

Corollary 3.2. With the above notation,

$$
\theta_{e}\left(t, O \tilde{R} \cup \bigcup_{j=1}^{m} D S C_{j}\right) \leqq \theta_{e}(t, R) \leqq \theta_{e}(t, \tilde{E} R)+\sum_{j=1}^{m} \theta_{e}\left(t, N S C_{j}\right)
$$

and there is a lower bound $\varrho$, depending only on the genus, for the eigenvalues in each sum as well as for $\theta(t, O \widetilde{R})$.

Proof. The left inequality follows from the right side of (3.1) and that the exclusion numbers are the same. The right inequality would also follow from (3.1) provided we knew that the first $2 m+q$ eigenvalues of $E \widetilde{R} \cup N S C$ were excluded. This presents no difficulty since any other exclusion produces a larger trace.

Now to consider the lower bound on the eigenvalues. The result is contained in the work of Cheng-Li, [36]; for the sake of exposition we shall give an alternate proof. The multiplicity of 0 in the spectrum of $\widetilde{R}$ is $q$, the number of components. Thus $\kappa_{q+1}(\widetilde{R})$ is positive and each eigenvalue depends continuously on the metric. By Lemma 2.3 there exists a positive lower bound $\beta$ for $\kappa_{q+1}(\widetilde{R})$. Now the 0 -mode of $\widetilde{R}$ occurs for $E \widetilde{R}$ and thus $\beta$ also bounds the $O \widetilde{R}$ spectrum. For the traces in the above inequalities the smallest eigenvalues occur on the right-hand side. In the next section we shall see that $\kappa_{3}\left(N S C_{j}\right)>\frac{1}{4}$ and it was noted above that $\kappa_{1}\left(D S C_{j}\right)>\frac{1}{4}$. Finally, the multiplicity of 0 in the spectrum of $E \widetilde{R}$ is simply the exclusion number. Thus $\beta$ is a lower bound for the spectrum of $E \widetilde{R}$. Set $\varrho=\min \left\{\beta, \frac{1}{4}\right\}$ and the proof is complete. 
3.5. The standard comparison of the Neumann and Dirichlet problems provides that $\theta(t, D S C) \leqq \theta(t, N S C)$. We shall now show a reverse inequality, $\theta_{e}(t, N S C)$ $\leqq \theta(t, D S C)+\theta_{1}(t)$, where $\theta_{1}$ is a fixed theta function. Our plan is to use the rotational symmetry to separate variables and reduce the problem to one dimension. Then we can use the interlacing of the Neumann and Dirichlet eigenvalues for a Sturm-Liouville problem.

The collar is a subdomain of the quotient $H /\left\langle z \rightarrow e^{\ell} z\right\rangle$. To simplify matters consider $w=u+i v=\log z$ and $H$ is mapped to the strip $\{0<v<\pi\}$; the transformation $z \rightarrow e^{\ell} z$ is represented as $w \rightarrow w+\ell$ and the hyperbolic metric is simply $\csc ^{2} v|d w|^{2}$. The subcollar $S C=\{2 \ell<\arg z<\pi-2 \ell\}$ is represented as $\{2 \ell<v<\pi-2 \ell\}$.

By separation of variables we write an eigenfunction as $f(v) \exp (2 \pi i k u / \ell)$, where $k$ is the rotational mode number. The Laplacian is $\sin ^{2} v\left(\frac{\partial^{2}}{\partial u^{2}}+\frac{\partial^{2}}{\partial v^{2}}\right)$ and the eigenvalue equation (recall $\kappa \geqq 0$ ) is

$$
\sin ^{2} v\left(f^{\prime \prime}(v)-(2 \pi k / \ell)^{2} f(v)\right)=-\kappa f(v)
$$

For NSC then $f$ has domain $[2 \ell, \pi-2 \ell]$ with $f^{\prime}$ vanishing at the end points; for DSC $f$ has the same domain but vanishes at the endpoints. For each fixed $k$ we have two Sturm-Liouville problems (1-dimensional, $2^{\text {nd }}$ order, selfadjoint) $N S C(k)$ and $D S C(k)$. A special feature of Sturm-Liouville is the (reverse) comparison

$$
\kappa_{n}(D S C(k)) \leqq \kappa_{n+2}(N S C(k)),[31]
$$

Roughly speaking the shift in index is explained by observing that a $\operatorname{DSC}(k)$ eigenfunction satisfies two linear equations: vanishing at the endpoints. We shall use this inequality to bound the Neumann spectrum below by the Dirichlet spectrum; all that remains is to estimate the first two eigenvalues of $N S C(k)$ from below.

To this end multiply (3.3) by $f(v) \csc ^{2} v$ and integrate by parts on $[2 \ell, \pi-2 \ell]$ to obtain

$$
\int f^{\prime}(v)^{2} d v+(2 \pi k / \ell)^{2} \int f^{2}(v) d v=\kappa \int f^{2}(v) \csc ^{2} v d v
$$

and trivially

$$
(2 \pi k)^{2} \int f^{2}(v) d v \leqq \ell^{2} \kappa \int f^{2}(v) \csc ^{2} v d v
$$

We shall now estimate the right-hand integral. The maximum of $\ell^{2} \csc ^{2} v$ on $[2 \ell, \pi-2 \ell]$ occurs at the endpoints where the value is approximately $\frac{1}{4}$ so $\ell^{2} \csc ^{2} v$ $\leqq \frac{1}{2}$ (for $\ell$ small). Substituting leaves $8(\pi k)^{2} \leqq \kappa$, the integrals having dropped out. (An upper bound $C k^{2}$ can be obtained by using a test function vanishing on [ $4 \ell, \pi$ $-4 \ell$ ] and rising linearly otherwise.) A lower bound for the first eigenvalue is also a bound for the higher eigenvalues. We have proven the following.

Corollary 3.3. For each $k \neq 0$,

$$
\theta(t, N S C(k)) \leqq \theta(t, D S C(k))+2 \exp \left(-8 \pi^{2} k^{2} t\right)
$$


We see now that the smallest eigenvalue of $N S C$ with a non-zero mode number is at least $8 \pi^{2}$. By $[11,30]$ the first two eigenvalues of $N S C$ are $<\frac{1}{4}$ (for $\ell$ small). Thus the exclusions for $\theta(t, N S C)$ are in the 0 rotational mode and by (3.4) $\kappa_{3}(N S C(0)) \geqq \kappa_{1}(D S C(0)) \geqq \kappa_{1}(D S C)>\frac{1}{4}$.

Definition 3.4.

$$
\theta_{1}(t)=\sum_{k=1}^{\infty} \exp \left(-8 \pi^{2} k^{2} t\right)
$$

Theorem 3.5. With the above notation,

$$
\theta_{e}(t, N S C) \leqq \theta(t, D S C)+4 \theta_{1}(t)
$$

Proof. Each rotational mode is treated separately. For the 0 -mode two eigenvalues are excluded from NSC and (3.4) provides the remaining estimate. For $k \neq 0$ the estimate is Corollary 3.3. The proof is complete.

We would like to point out two senses in which $\theta_{1}$ is small. First $\theta_{1}$ is independent of $\ell$, the length of the core geodesic. Next if we consider $\theta_{1}$ as a heat trace then the spectrum $\lambda_{k} \sim c k^{2}$ is that of a 1 -dimensional problem.

3.6. Recall the lower bound $\varrho$ of Corollary 3.2 .

Theorem 3.6. With the above notation,

$$
\begin{aligned}
\theta(t, O \widetilde{R}) & +\sum_{j=1}^{m} \theta\left(t, D S C_{j}\right)-(2 m+q) e^{-e t} \leqq \theta_{e}(t, R) \\
& \leqq \theta_{e}(t, E \widetilde{R})+\sum_{j=1}^{m} \theta\left(t, D S C_{j}\right)+4 m \theta_{1}(t)
\end{aligned}
$$

Furthermore, the lower bound and upper bound satisfy $B(t) \sim$ Area $(R) / 4 \pi t$ for small $t$ (but not uniformly in $R$ ).

Proof. The right side is Corollary 3.2 and Theorem 3.5. The left side follows from Corollary 3.2. In particular the spectrum for $O \widetilde{R} \cup \bigcup_{j} D S C_{j}$ is bounded below by $\varrho$, thus each of the $2 m+q$ excluded terms is bounded above by $e^{-\varrho t}$. The inequality is now clear. Finally, we consider the small time asymptotics. Recall that $\theta_{1}(t) \sim c^{/} \sqrt{t}$ for small $t$ and thus this term is lower order. By Weyl's asymptotic law the problem is reduced to finding the area for each term. Now for the spectrum $O \widetilde{R}=D(R-S C)$ and $E \widetilde{R}=N(R-S C)$, and thus each of these problems has the area of $R-S C$. The conclusion now follows from Area $(R-S C)+$ Area $(S C)=$ Area $(R)$.

Remark. The key feature of the final estimate is that the Dirichlet spectrum appears on both sides of the inequality.

\section{Estimates for the Trace of the Heat Kernel}

4.1. The small eigenvalues have been removed from the heat trace $\theta_{e}(t)$ to obtain an expression for the Selberg zeta function $Z(s)$ valid for $s$ near 1. The next step is to find the divergence of the trace $\theta_{e}(t)$ for $\ell \rightarrow 0$. By the Trace Formula $\theta(t)$ is a sum of 
contributions, one for each geodesic. We shall show that the $\ell$-divergence $\theta_{e}(t) \approx \frac{e^{-t / 4}}{\sqrt{\pi t}} \log \frac{1}{\ell}$, as $\ell \rightarrow 0$, is as simple as possible; the sum of the contributions for the non-short geodesics is uniformly bounded. Actually this is not quite sufficient. We need that $\theta_{e}(t)$ is the sum of the contributions of the short geodesics and a term of order $O\left(e^{-\varepsilon t}\right)$, for $\varepsilon>0, \varepsilon$ independent of $R, t \geqq 1$.

By the estimate of Cheng (see Chap. 1, Sect. 4) one knows ahead of time that the divergence of $\theta_{e}(t)$ is due to the accumulation of eigenvalues near $\frac{1}{4}$. Now $\theta(t)$ is the Laplace transform of the spectral counting function $N(x)=\#\left\{\kappa_{n} \leqq x\right\}$. As such the small $x$ behavior if $N(x)$ is measured by the large $t$ behavior of $\theta(t)$. Specifically the large $t$-behavior of $\sum_{\kappa_{n} \geqq \frac{1}{4}} e^{-\kappa_{n} t}$ is the transform of the accumulation of eigenvalues at $\frac{1}{4}$.

By Theorem 3.6 the problem is now reduced to the case of a subcollar with Dirichlet boundary data. This is an ideal situation: the spectrum starts above $\frac{1}{4}$, [11], and as we shall see in Sects. 4.2 and 4.3 the spectral divergence is due to the rotationally invariant functions. In Sect. 4.4 we combine our arguments to obtain the final estimate for $\theta_{e}(t)$. In Sect. 4.5 we compare the spectral divergence for a hyperbolic surface to that of a Euclidean cylinder and also we comment on Cheng's estimate.

4.2. We would like to give an upper bound for $\theta(t, D S C)$. The heat kernel is not known explicitly and thus we shall use a comparison kernel. Recall that a subcollar is a subdomain of the quotient $H /\left\langle z \rightarrow e^{\ell} z\right\rangle$ and that the heat kernel of a domain is an upper bound for the Dirichlet heat kernel of any subdomain. We shall use the heat kernel of the quotient as an upper bound for the subcollar.

Let $\Gamma_{\ell}$ be the group generated by $z \rightarrow e^{\ell} z$ and

$$
k(z, w ; t)=k(\delta(z, w) ; t)=\frac{\sqrt{2} e^{-t / 4}}{(4 \pi t)^{3 / 2}} \int_{\delta}^{\infty} \frac{e^{-b^{2} / 4 t} b d b}{\sqrt{\operatorname{chb}-\operatorname{ch} \delta}}
$$

$[24$, p. 233] the elementary solution of the heat equation, where $\delta(z, w)$ is the distance from $z$ to $w$. By the standard argument $K_{\ell}(z, w ; t)=\sum_{n=-\infty}^{\infty} k\left(z, e^{n \ell} w ; t\right)$ converges and is a $\Gamma_{\ell}$ invariant elementary solution, [14]. Denote by $K S C(z, w ; t)$ both the heat kernel of $D S C$ (Dirichlet problem for the subcollar) as well as its lift to the universal cover $S \widetilde{C} \subset H$. For $w$ fixed $\left(K_{\ell}-K S C\right)(z, w ; 0)$ vanishes and for $t \neq 0$, $z \in \partial S C,\left(K_{\ell}-K S C\right)(z, w ; t)$ is positive, since $K_{\ell}$ is a positive sum and $K S C$ vanishes. Thus by the maximum principle $K_{\ell} \geqq K S C$.

In Sect. 5.2 we shall recall the formula for $\theta(t, R)$ in terms of the lengths of closed geodesics (Selberg trace formula). Let $T(t, \ell)$ be the total contribution to $\theta(t)$ by a primitive nonoriented geodesic of length $\ell$.

Definition 4.1. $T(t, \ell)=\frac{1}{2} \sum_{n=1}^{\infty} \ell \operatorname{csch} \frac{\ell n}{2} \frac{e^{-t / 4}}{\sqrt{\pi t}} e^{-\ell^{2} n^{2} / 4 t}$

We shall see that the divergence of $\theta(t)$ is due to the contribution of the short geodesics. The behavior of $T(t, \ell)$ for $\ell$ small is given by comparing the sum to 
the integral

$$
\frac{e^{-t / 4}}{2 \sqrt{\pi t}} \int_{\ell}^{\infty} \operatorname{csch} \frac{x}{2} \mathrm{e}^{-x^{2} / 4 t} d x=\frac{e^{-t / 4}}{2 \sqrt{\pi t}}\left(\int_{\ell}^{1} \operatorname{csch} \frac{x}{2} e^{-x^{2} / 4 t} d x+O(1)\right) .
$$

For $t \geqq 1$ the integral $\frac{1}{2} \int_{\ell}^{1} \operatorname{csch} \frac{x}{2} e^{-x^{2} / 4 t} d x$ is $\log \frac{1}{\ell}+O(1)$, and in particular $T(t, \ell)=\frac{e^{-t / 4}}{\sqrt{\pi t}}\left(\log \frac{1}{\ell}+O(1)\right)$ uniformly for $t \geqq 1$ and $\ell \leqq c_{*}$. This will be our upper bound for the divergence.

Lemma 4.2. $\theta(t, D S C) \leqq k(0 ; t)$ Area $(S C)+T(t, \ell)$.

Proof. As already noted, an upper bound is given by $\int_{S C} K_{\ell}(z, z ; t) d A$. Substituting the sum for $K_{\ell}$, the $n=0$ terms is $k(0 ; t)$ Area $(S C)$ and for the remaining terms (all positive) we may replace $S C$ with $H / \Gamma_{\ell}$. The resulting sum of integrals is exactly the contribution of a length $\ell$ geodesic for the Selberg trace formula, [18].

Remark. So that the reader may compare $T(t, \ell)$, estimated above, with $k(0 ; t)$ we recall that $k(0 ; t)$ is $\frac{g-1}{t}+O(1)$ for small $t$ and $\frac{O\left(e^{-t / 4}\right)}{t^{3 / 2}}$ for $t$ large.

4.3. By the above discussion the $\ell$-divergence is at most $\frac{e^{-t / 4}}{\sqrt{\pi t}} \log \frac{1}{\ell}$. Now we shall see that the spectral divergence for the rotationally invariant functions is bounded below by the same quantity.

We return to the discussion of Sect. 3.5 and the formula (3.3) for $k=0$,

$$
\sin ^{2} v f^{\prime \prime}(v)=-\kappa f(v)
$$

on the interval $[2 \ell, \pi-2 \ell]$ with Dirichlet boundary boundary conditions. The spectrum of this problem is bounded above by the union of that for $\left[2 \ell, \frac{\pi}{2}\right]$ and $\left[\frac{\pi}{2}, \pi-2 \ell\right]$, each with Dirichlet boundary conditions. By the symmetry we have with the obvious notation $2 \theta\left(t,\left[2 \ell, \frac{\pi}{2}\right]\right) \leqq \theta(t,[2 \ell, \pi-2 \ell])$.

The variational quotient for (3.3) is

$$
\int f^{\prime}(v)^{2} d v / \int \csc ^{2} v f(v)^{2} d v
$$

To estimate the eigenvalues above we may replace $\csc ^{2} v$ by the smaller weight $v^{-2}$. Actually this comparison is geometrically natural; for our problem the eigenfunctions are invariant by a family of 1-dimensional hyperbolic subgroups of $S L(2 ; \mathbb{R})$ which are conjugated to converge to a 1-dimensional parabolic subgroup.

To summarize the above a lower bound for $\theta\left(t,\left[2 \ell, \frac{\pi}{2}\right]\right)$ is given by twice the 
trace of the Dirichlet problem on $\left[2 \ell, \frac{\pi}{2}\right]$ of

$$
v^{2} f^{\prime \prime}(v)=-\kappa f(v) \text {. }
$$

This is a standard eigenvalue problem for $S L(2 ; \mathbb{R})$; to recall the solution first note that the equation is preserved by the substitution $v=m \tilde{v}$. The interval may be replaced by $[1, \pi / 4 \ell]$. Now writing $\kappa$ as $\kappa=\frac{1}{4}+r^{2}$ the general solution is $\varphi(v)=a v^{\frac{1}{2}+i r}+b v^{\frac{1}{2}-i r}$ for $r \neq 0$ and $\varphi(v)=a v^{\frac{1}{2}}+b v^{\frac{1}{2}} \log v$ for $r=0$. The solutions for the boundary conditions are:

$$
\varphi(v)=v^{\frac{1}{2}} \sin \left(\alpha_{n} \log v\right) \quad \text { for } \quad \alpha_{n}=n \pi / \log (\pi / 4 \ell), n \geqq 1 .
$$

The eigenvalues are $\kappa_{n}=\frac{1}{4}+\alpha_{n}^{2}$ for $n \geqq 1$.

Lemma 4.3. With the above notation,

$$
\theta(t,[2 \ell, \pi-2 \ell]) \geqq e^{-t / 4}\left(\frac{\log \pi / 4 \ell}{\sqrt{\pi t}}-1\right) .
$$

Proof. We set $A=\pi /(\log \pi / 4 \ell)^{2}$ and summarize the above discussion with $\theta(t,[2 \ell, \pi-2 \ell]) \geqq 2 e^{-t / 4} \sum_{n=1}^{\infty} e^{-\pi n^{2} A t}$. By the Jacobi inversion formula the last quantity is $e^{-t / 4}\left(\frac{1}{\sqrt{A t}} \sum_{n=-\infty}^{\infty} e^{-\pi n^{2} / A t}-1\right)$. We estimate below by the $n=0$ term, the proof is complete.

4.4. As the first step towards estimating $Z(s)$ we now consider the Laplace transform of $\theta_{e}(t)$. We introduce appropriate notation,

$$
\begin{aligned}
I(t) & =\theta_{e}(t, R)-k(0 ; t) \text { Area }(R), \\
J(\xi) & =\int_{0}^{\infty} e^{-\xi t} I(t) d t
\end{aligned}
$$

and recall the eigenvalue bound $\varrho$ of Corollary 3.2. Our estimate is for a surface $R$ with short geodesics (length $\leqq c_{*}$ ) of lengths $\left\{\ell_{j}\right\}_{j=1}^{m}$.

Theorem 4.4. With the above notation, given $\varepsilon>0$ there exists a constant $\beta$, depending only on $\varepsilon$ and the genus, such that

$$
\left|J(\xi)-\sum_{j=1}^{m} \int_{0}^{\infty} e^{-\xi t} T\left(t, \ell_{j}\right) d t\right| \leqq \beta
$$

for $\operatorname{Re} \xi \geqq-\varrho+\varepsilon$.

Proof. It suffices to bound the integral $\int e^{-\xi t}\left|I(t)-\sum_{j} T\left(t, \ell_{j}\right)\right| d t, \xi$ real. Equivalently we may give upper and lower bounds for the integrand $I(t)-\sum_{j} T\left(t, \ell_{j}\right)$, provided of course that the maximum (for each $t$ ) of the bounds itself has a bounded Laplace transform. 
We start with a lower bound for the integrand. $I(t)$ is bounded below by $\theta(t)-k(0 ; t)$ Area $-e_{*}$, where $e_{*}$ is the exclusion number of Sect. 3.4 and thus is integrable for $t$ small. This is also our first estimate, $I(t) \geqq \theta(t)-k(0 ; t)$ Area $-e_{*}$ for $0 \leqq t \leqq 1$. We wish to compare $I(t)$ with the length sum for $\theta(t)$ given by the Selberg trace formula. Since each length term is positive, a lower bound is obtained by omitting the terms for non-short geodesics. To summarize for $0 \leqq t \leqq 1$

$$
I(t) \geqq \theta(t)-k(0 ; t) \text { Area }-e_{*} \geqq \sum_{j=1}^{m} T\left(t, \ell_{j}\right)-e_{*},
$$

our final estimate for this situation.

Now we must also give a suitable lower bound for $I(t), 1 \leqq t<\infty$. The first step is to apply Theorem 3.6. We can omit the positive term $\theta(t, O \widetilde{R})$. The trace is estimated by Lemma 4.3. To summarize thus far

$$
I(t) \geqq \sum_{j} e^{-t / 4}\left(\frac{\log \pi / 4 \ell_{j}}{\sqrt{\pi \mathrm{t}}}-1\right)-(2 m+q) e^{-\varrho t} \text { for } t \geqq 1 .
$$

The next step is to replace the $\log 1 / \ell$ term by $T(t, \ell)$. By integral comparison,

$$
0 \leqq \sqrt{\pi t} e^{t / 4} T(t, \ell)-\frac{1}{2} \int_{\ell}^{\infty} \operatorname{csch} \frac{x}{2} e^{-x^{2} / 4 t} d x \leqq \frac{1}{2} \ell \operatorname{csch} \frac{\ell}{2} e^{-\ell^{2} / 4 t}
$$

After simplifications we see that

$$
\sqrt{\pi t} e^{t / 4} T(t, \ell)-\frac{1}{2} \int_{\ell}^{1} \operatorname{csch} \frac{x}{2} e^{-x^{2} / 4 t} d x \quad \text { is } \quad O(1) \quad \text { for } t \geqq 1
$$

and also that

$$
\frac{1}{2} \int_{\ell}^{1} \operatorname{csch} \frac{x}{2} e^{-x^{2} / 4 t} d x-\log 1 / \ell \quad \text { is } \quad O(1) \quad \text { for } t \geqq 1 .
$$

To summarize these remarks there exists a constant $c_{1}$ such that

$$
I(t) \geqq \sum_{j=1}^{m} T\left(t, \ell_{j}\right)-c_{1} e^{-t / 4}-(2 m+q) e^{-\varrho t} .
$$

We recall that $m$ and $q$ are bounded in terms of the genus. Combining (4.2) and (4.3) we have that there exists a constant $c_{2}$ such that

$$
I(t) \geqq \sum_{j=1}^{m} T\left(t, \ell_{j}\right)-c_{2} e^{-\varrho t} .
$$

Our discussion of lower bounds for $I(t)$ is complete.

Now we consider the upper bounds for $I(t)$. The estimate is obtained by combining Theorem 3.6 and Lemma 4.2. We have the inequality

$$
\begin{aligned}
I(t) \leqq & \theta_{e}(t, E \widetilde{R})+k(0 ; t)\left(\sum_{j=1}^{m} \operatorname{Area}\left(S C_{j}\right)-\operatorname{Area}(R)\right) \\
& +\sum_{j=1}^{m} T\left(t, \ell_{j}\right)+4 m \theta_{1}(t) .
\end{aligned}
$$


By the standard small time expansion the right-hand side is integrable for small $t$, [41]. By Corollary 3.2. $\theta_{e}(t, E \widetilde{R})$ is $O\left(e^{-o t}\right)$ for $t$ large and each remaining term is $O\left(e^{-t / 4}\right)$. Inequality (4.5) is our upper bound.

To summarize the discussion to this point we have for $I(t)=\theta_{e}(t, R)$ $-k(0 ; t)$ Area $(R)$ that there exists a constant $c_{3}$ with

$$
\begin{aligned}
& \left|I(t)-\sum_{j=1}^{m} T\left(t, \ell_{j}\right)\right| \\
& \quad \leqq \mid \theta_{e}(t, E \tilde{R})-\frac{1}{2} k(0 ; t) \text { Area }(\tilde{R}) \mid+4 m \theta_{1}(t)+c_{3} e^{-e t} .
\end{aligned}
$$

This is simply the statement that the right-hand side dominates the bounds of (4.4) and (4.5). The $\theta_{1}(t)$ contribution is certainly bounded: $\theta_{1}(t) \sim c / \sqrt{t}$ for small $t$ and is $O\left(e^{-8 \pi^{2} t}\right)$ for large $t$. The final step is to bound the $E \tilde{R}$ contribution.

McKean and Singer treat the small time expansion of the heat trace for manifolds with boundary (as well as their doubles), [41, esp. Sects. 3 and 5]. They note that the elementary solution of the heat equation can be expressed as an infinite sum (their formula 3.4) by the method of Levi. The convergence estimates for the sum only require simple estimates for the Riemannian metric; the constants can be chosen to be uniform for a compact family of Riemannian manifolds with boundary. Thus from their treatment and the concluding comments of Sect. 2.6, $\theta(t, E \tilde{R})-\frac{\text { Area }(E \tilde{R})}{4 \pi t}$ is uniformly bounded for $t$ bounded. This is enough to bound $\int e^{-\xi t} \mid \theta_{e}(t, E \tilde{R})-\frac{1}{2} k(0 ; t)$ Area $(\widetilde{R}) \mid d t$ for $\operatorname{Re} \xi>-\varrho$, since $\theta_{e}(t, E \tilde{R})$ $\leqq e^{-\varrho t} \theta_{e}(1, E \tilde{R})$ for $t \geqq 1, k(0 ; t)-\frac{1}{4 \pi t}$ is $O(1)$ for small $t$ and $k(0 ; t)$ is $O\left(e^{-t / 4}\right)$ for large $t$. This is the desired final estimate: given $\varepsilon>0$ the $E \tilde{R}$ contribution is bounded for $\xi$ real, $\xi \geqq-\varrho+\varepsilon$. The proof is complete.

4.5. We would like to close this chapter with a heuristic discussion of the convergence of the spectral measure of $R$. Specifically if $N(x, R)=$ \# \{eigenvalues of $R \leqq x\}$, then we shall see (no proof) that

$$
N(x, R) / \sum_{j} 2 \log 1 / \ell_{j} \rightarrow \frac{1}{\pi} \sqrt{x-\frac{1}{4}} .
$$

Recall that $2 \log 1 / \ell$ is essentially the width of a collar. In fact for the case of one separating short geodesic on a compact surface then $0 \leqq \operatorname{diameter}(R)$ $-2 \log 1 / \ell \leqq c$. By comparison the eigenvalue estimate of S. Y. Cheng is $\kappa_{n+1} \leqq \frac{1}{4}+\left(\frac{4 \pi n}{\operatorname{diam}}\right)^{2},[8]$

Our main result (estimate (4.6)) is of the form

$$
\theta(t) / \sum_{j} \log 1 / \ell_{j} \rightarrow \frac{e^{-t / 4}}{\sqrt{\pi t}}
$$

or by a Laplace transform $d N(x, R) / \sum_{j} \log 1 / \ell_{j} \rightarrow \frac{d x}{\pi \sqrt{x-\frac{1}{4}}}$ (actually we can 
establish this convergence weak* relative to $\left.C_{c}(0, \infty)\right)$. We may formally integrate to obtain $N(x, R) / \sum_{j} 2 \log 1 / \ell_{j} \rightarrow \frac{1}{\pi} \sqrt{x-\frac{1}{4}}$. Now Cheng's estimate gives the (surprisingly close) lower bound $N(x, R) \geqq \frac{\text { diam }}{4 \pi} \sqrt{x-\frac{1}{4}}$. We shall comment further on this below. Cheng's argument is by constructing test functions for the RayleighRitz quotient.

Inspired by the paper of Gava-Iengo-Jayaraman-Ramachandran, [17] and also the description of tachyon divergence we would like to compare the hyperbolic case to the Euclidean case. Consider the flat torus obtained by identifying the sides of a rectangle of length $1 / \varepsilon$ and width $\varepsilon$. The eigenvalues are $\kappa_{n, m}=(2 \mathrm{~m} \pi \varepsilon)^{2}+(2 \mathrm{n} \pi / \varepsilon)^{2}$ and the counting function $N_{\varepsilon}(x) \approx \frac{\sqrt{x}}{\pi \varepsilon}$ for $x$ fixed and $\varepsilon \rightarrow 0$. There is an obvious analogy with the above.

Actually, this represents a simpler phenomenon than tachyon divergence. This result is for the trace of the heat kernel and not the bosonic Polyakov integrand. The analogy between the spectral divergences of the two geometries can even be refined. By the considerations of Sects. 4.2 and 4.3 the divergence is for the rotationally invariant eigenfunctions. The corresponding result for a torus or cylinder is obvious. The divergence is for those waves (eigenfunctions) which propagate across the cylinder but are constant on the meridians. Cheng's test functions are essentially for these modes of oscillation.

\section{Estimates for the Selberg Zeta Function}

5.1. McKean's observation is that the Selberg zeta function is obtained easily from the trace $\theta(t)$ of the heat kernel,

$$
\frac{Z^{\prime}(s)}{Z(s)}=(2 s-1) \int_{0}^{\infty} e^{-\xi t}(\theta(t)-k(0 ; t) \text { Area }) d t
$$

for $\xi=s(s-1), \operatorname{Re} \xi>0$. Our plan is quite simple. Naively the integral converges in the largest $\xi$-half plane where the (analytic continuation of the) transform is pole free. By subtracting those terms from the integrand (the contributions of the small eigenvalues) which give rise to the poles, we can obtain an integral convergent in a larger half plane. Of course the whole problem is to estimate the new integrand $\left(\theta_{e}(t)-k(0 ; t)\right.$ Area); our estimates are Theorem 3.6 and the inequality (4.6).

We start by reviewing the formulas for $\theta(t)$ and $Z(s)$. In the third section we review the divergence of $\mathscr{Z}(s, \ell)$ as $\ell \rightarrow 0$. We show in Sect. 5.4 that the length product (for $Z(s)$ ), $\operatorname{Re} s>1$, for the non-short geodesics is uniformly bounded. In Sect. 5.5 our estimates are combined to show that

$$
B^{-1} \leqq Z^{\prime}(1) / \prod_{j=1}^{m} \exp \left(-\pi^{2} / 3 \ell_{j}\right) \ell_{j}^{-1} \prod_{0<\kappa_{n}<\frac{1}{4}} \kappa_{n} \leqq B
$$

where $B$ depends only on the genus and $\left\{\ell_{j}\right\}$ are the short geodesic lengths. The next 
item is a remark on extending our results to the left of $\operatorname{Re} s=\frac{1}{2}$. Then in the final section we consider the convergence of the length spectrum and of the Selberg zeta function to that of the limiting surface.

5.2. Of course we start with the trace formula for the heat kernel. Consider $R$ a compact surface with eigenvalue spectrum $\left\{\kappa_{n}\right\}(\kappa \geqq 0)$. We write $\sum_{\gamma}$ for a sum over the closed primitive nonoriented geodescis of $R$ and write $\ell(\gamma)$ for the length of $\gamma$. The Trace Formula is

$$
\theta(t)=k(0 ; t) \text { Area }+\frac{1}{2} \sum_{\gamma} \sum_{n=1}^{\infty} \ell(\gamma) \operatorname{csch} \frac{\ell(\gamma) n}{2} \frac{e^{-t / 4} e^{-\ell(\gamma)^{2} n^{2} / 4 t}}{\sqrt{\pi t}},
$$

where of course the second term is $\sum_{\gamma} T(t, \ell(\gamma))$ in the notation of Sect. 4.2, [18, 38]. The Selberg zeta function is defined by the infinite product

$$
Z(s)=\prod_{\gamma} \prod_{k=0}^{\infty}\left(1-e^{-(s+k) \ell(\gamma)}\right)^{2} \quad \text { for } \quad \operatorname{Re} s>1
$$

(each geodesic corresponds to two conjugacy classes, one for each orientation). The basic properties of $Z(s)$ are discussed in [18]. We shall only need that $Z(s)$ is an entire function, has the symmetry,

$$
Z(s)=\chi(s) Z(1-s), \quad \chi(s)=\exp \left[\text { Area } \int_{0}^{s-\frac{1}{2}} v \tan (\pi v) d v\right],
$$

and has zeros corresponding to eigenvalues $\left\{\kappa_{n}\right\}$ such that the formal quotient $Z(s) / \Pi\left(s^{2}-s+\kappa_{n}\right)$ is pole free. As an example $Z(s) / \prod_{0 \leqq \kappa_{n}<\frac{1}{4}}\left(s^{2}-s+\kappa_{n}\right)$ is analytic and non-zero in the half plane $\operatorname{Re} s>\frac{1}{2}$.

Finally, we would like to recall the formula of McKean

$$
\frac{Z^{\prime}(s)}{Z(s)}=(2 s-1) \int_{0}^{\infty} e^{-s(s-1) t}(\theta(t)-k(0 ; t) \text { Area }) d t
$$

for $\operatorname{Re} s(s-1)>1$, [24]. We shall sketch the derivation of this formula (the formulas from this reference should be double checked). By Weyl's law the integrand is integrable for small $t$ and trivially $\theta(t)$ is $O(1)$ for large $t$. Substituting the length expansion of $\theta(t)$ leaves

$$
(2 s-1) \int_{0}^{\infty} e^{-s(s-1) t} \sum_{\gamma} T(t, \ell) d t ;
$$

the sum is positive and we may integrate term by term. The Laplace transform of $T(t, \ell)$ is in the standard tables.

5.3. The contribution to $Z(s)$ of a geodesic of length $\ell$ is the elementary product

$$
\mathscr{Z}(s)=\prod_{k=0}^{\infty}\left(1-e^{-(s+k) \ell}\right)^{2} .
$$

We would like to review the divergence behavior of $\mathscr{Z}(s)$ as $\ell \rightarrow 0$. 
Lemma 5.1. Given a compact subset $K$ of $\{\operatorname{Re} s>0\}$ there exists a positive constant $c_{K}$ such that

$$
c_{K}^{-1} \leqq\left|\mathscr{Z}(s) e^{\pi^{2} / 3 \ell} \ell^{2 s-1}\right| \leqq c_{K}
$$

for $s \in K$ and $\ell<1$.

Proof. By elementary expansion

$$
-\log \prod_{k=0}^{\infty}\left(1-e^{-(s+k) \ell}\right)=\sum_{n=1}^{\infty} \sum_{k=0}^{\infty} \frac{e^{-n(s+k) \ell}}{n}=\sum_{n=1}^{\infty} e^{-n s \ell} / n\left(1-e^{-n \ell}\right) .
$$

Now $\left|\frac{1}{1-e^{-x}}-\frac{1}{x}-\frac{1}{2}\right| \leqq a x$ for $x \geqq 0$, and thus the error on replacing $\frac{1}{1-e^{-n \ell}}$ by $\frac{1}{n \ell}+\frac{1}{2}$ is at most $\sum_{n=1}^{\infty} \frac{e^{-n s \ell}}{n}$ an $\ell=\frac{\ell e^{-s \ell}}{\left(1-e^{-s \ell}\right)}$, which is uniformly bounded for $\ell$ small and $s$ bounded away from 0 . Now the $\frac{1}{n \ell}$ contribution is

$$
\begin{aligned}
\sum_{n=1}^{\infty} \frac{e^{-n s \ell}}{n^{2} \ell} & =-\frac{1}{\ell} \int_{0}^{e^{-s \ell}} \frac{1}{t} \log (1-t) d t \\
& =\frac{\pi^{2}}{6 \ell}+\frac{1}{\ell} \int_{e^{-s \ell}}^{1} \frac{1}{t} \log (1-t) d t \\
& =\frac{\pi^{2}}{6 \ell}+s \log \left(1-e^{-s \ell}\right)+\frac{1}{\ell} \int_{e^{-s \ell}}^{1} \frac{\log t}{1-t} d t,
\end{aligned}
$$

where the integration path of the final integral is defined such that $\log e^{-s \ell}=-s \ell$ and $\log 1=0$, and thus the last term is bounded uniformly for $s \ell$ small. And finally, the $\frac{1}{2}$ contribution is $\frac{1}{2} \sum_{n=1}^{\infty} \frac{e^{-n s \ell}}{n}=-\frac{1}{2} \log \left(1-e^{-s \ell}\right)$, the conclusion now follows.

5.4. Now we consider the partial product for the non-short geodesics

$$
Z_{*}(s)=\prod_{\substack{\gamma \\ \ell(\gamma) \geqq c_{*}}} \prod_{k=0}^{\infty}\left(1-e^{-(s+k) \ell(\gamma)}\right)^{2} \text { for } \operatorname{Re} s>1 .
$$

The simple structure of $Z(s)$ will allow us to estimate $Z_{*}(s)$ without integrating $Z^{\prime} \mid Z$.

Theorem 5.2. Given a compact subset $K$ of $\{\operatorname{Re} s>1\}$, there exists a positive constant $A$ depending only on the genus such that

for $s \in K$.

$$
A^{-1} \leqq\left|Z_{*}(s)\right| \leqq A
$$

Proof. The argument is illustrated for the case of $s$ real. We shall bound $\left|\log Z_{*}(s)\right|$. By a simple comparison it is enough to bound $\sum_{\substack{\gamma \\ \ell(\gamma) \geqq c_{*}}} \sum_{k=0}^{\infty} e^{-(s+k) \ell(\gamma)}$. In fact since 
$\ell(\gamma) \geqq c_{*}$ it will certainly be enough if we bound

$$
2 \sum_{\substack{\gamma \\ \ell(\gamma) \geqq c_{*}}} \sum_{k=0}^{\infty} \frac{\ell(\gamma) e^{-(s+k) \ell(\gamma)}}{1-e^{-(s+k) \ell(\gamma)}}=\frac{Z_{*}^{\prime}(s)}{Z_{*}(s)} .
$$

But by McKean's formula this is equivalent to bounding

$$
(2 s-1) \int_{0}^{\infty} e^{-\xi t} \sum_{\substack{\gamma \\ \ell(\gamma) \geqq c_{*}}} T(t, \ell(\gamma)) d t
$$

Now since $\operatorname{Re} s>1$ and $\operatorname{Re} \xi>0$, such a bound follows from Theorem 4.4 after an estimate for the contribution of the small eigenvalues.

5.5 By analogy with the heat trace we expect $Z(s)$ to be uniformly bounded after removing the contribution of the small eigenvalues and the short geodesics.

Theorem 5.3. There exists a positive constant depending only on the genus such that

$$
B^{-1} \leqq Z^{\prime}(1) / \prod_{j=1}^{m} \exp \left(-\pi^{2} / 3 \ell_{j}\right) \ell_{j}^{-1} \prod_{0<\kappa_{n}<\frac{1}{4}} \kappa_{n} \leqq B
$$

where $\left\{\ell_{j}\right\}_{j=1}^{m}$ are the short geodesic lengths.

Proof. The first step is to change to an equivalent expression. By Lemma 5.1 a factor $\exp \left(-\pi^{2} / 3 \ell\right) \ell^{-1}$ can be replaced by $\mathscr{Z}(1, \ell)$. Similarly by Sect. 3.2, $\prod_{0<\kappa_{n}<\frac{1}{4}} \kappa_{n}$ can be replaced by $\prod_{1<n<2 m+q} \kappa_{n}$. In particular if $\kappa_{j}$ is small, then the surface has a short $j$-disconnect and by definition $q \geqq j$, thus a small factor in the first eigenvalue product appears in the second. Furthermore, the number of factors is bounded in terms of the genus (recall $\kappa_{4 g-1} \geqq \frac{1}{4}$ ) and there are uniform bounds for the $\kappa_{j}, j \geqq 2 g-1$. If we introduce the product $Q(s)=\prod_{n=1}^{2 m+q}\left(s^{2}-s+\kappa_{n}\right)$, where $Q(1)=0$ and $Q^{\prime}(1)=2 \prod_{n=1}^{2 m+q} \kappa_{n}$, then for $Z_{* *}(s)=Z_{*}(s) / Q(s)$, we have $Z^{\prime}(1)=Z_{* *}(1) Q^{\prime}(1) \prod_{j=1}^{m} \mathscr{Z}\left(1, \ell_{j}\right)$. And so now the object is to estimate $Z_{* *}(1)$.

Our plan is to first estimate $Z_{* *}^{\prime}(s) / Z_{* *}(s)$ for $s$ real, $s \geqq 1$ and then integrate. By definition

and

$$
\frac{Z^{\prime}(s)}{Z(s)}=\frac{Z_{* *}^{\prime}(s)}{Z_{* *}(s)}+\sum_{j=1}^{m} \frac{\mathscr{Z}^{\prime}\left(s, \ell_{j}\right)}{\mathscr{Z}\left(s, \ell_{j}\right)}+\frac{Q^{\prime}(s)}{Q(s)}
$$

$$
\begin{gathered}
(2 s-1) \int_{0}^{\infty} e^{-(\xi+\kappa) t} d t=\frac{2 s-1}{s^{2}-s+\kappa}, \\
(2 s-1) \int_{0}^{\infty} e^{-\xi t} T(t, \ell) d t=\frac{\mathscr{Z}^{\prime}(s, \ell)}{\mathscr{Z}(s, \ell)} .
\end{gathered}
$$


Thus for the present context McKean's formula is (and this is the key)

$$
\frac{Z_{* *}^{\prime}(s)}{Z_{* *}(s)}=(2 s-1) \int_{0}^{\infty} e^{-\xi t}\left(\theta_{e}(t)-k(0 ; t) \text { Area }-\sum_{j=1}^{m} T\left(t, \ell_{j}\right)\right) d t
$$

At first sight the formula is valid for $\operatorname{Re} \xi>0, \xi=s(s-1)$. But by Theorem 4.4 the right-hand side is holomorphic in a small neighborhood of $\xi=0$ (hence $s=1$ ) and also there exists a positive constant $\beta$ such that

$$
\beta^{-1} \leqq\left|\frac{Z_{* *}^{\prime}(s)}{Z_{* *}(s)}\right| \leqq \beta \quad \text { for } s \text { real, } \quad s \geqq 1 .
$$

Now we must in effect estimate the constant of integration, which we choose to be $Z_{* *}(2) . Q(2)$ is uniformly bounded and thus by Theorem 5.2 so is $Z_{* *}(2)$. Finally, we write $\log Z_{* *}(1)=\int_{2}^{1} Z_{* *}^{\prime}(s) / Z_{* *}(s) d s+\log Z_{* *}(2)$, and the desired estimate follows. The proof is complete.

Corollary 5.4. Let $\varrho$ be the bound of Corollary 3.2. $\left|Z_{* *}(s)\right|$ is uniformly bounded above and below on compact subsets of $\left\{\operatorname{Re}\left(s^{2}-s\right)>-\varrho\right\}$.

Proof. Combine (5.1) with Theorem 4.4.

5.6. The quotient $Z_{*}(s)=Z(s) / \prod_{j=1}^{m} \mathscr{Z}\left(s, \ell_{j}\right)$ (see Sects. 5.3, 5.4 for notation) cannot converge for $\operatorname{Re} s<\frac{1}{2}$ as $\ell_{j} \rightarrow 0$. To see this we exploit the symmetry of the Selberg zeta function

$$
Z(s) / \mathscr{Z}(s)=\chi(s) Z(1-s) / \mathscr{Z}(s)=\chi(s)(Z(1-s) / \mathscr{Z}(1-s)) \mathscr{Z}(1-s) / \mathscr{Z}(s),
$$

where by Lemma $5.1 \mathscr{Z}(1-s) / \mathscr{Z}(s) \approx \prod_{j=1}^{m} \ell_{j}^{4 s-2}$. Simply observe that for $0<s<\frac{1}{2}$ and $\ell_{j} \rightarrow 0$ each factor $\ell_{j}^{4 s-2}$ is divergent.

5.7. We would like to return to the context of Sect. 2.5 , where we considered a parametrized family of smooth Riemann surfaces $R_{\tau}, \tau \in \mathbb{C}^{n}$ converging to a noded surface $R_{0}$. Let $\ell_{1}, \ldots, \ell_{m}$ be the lengths of geodesics which are tending to zero as $\tau \rightarrow 0$ and $S_{1}, \ldots, S_{q}$ the components of $R_{o}$-\{nodes\}. Define $Z\left(s, R_{o}\right)=\prod_{n=1}^{q} Z\left(s, S_{n}\right)$, i.e. the product of the zeta functions of the components.

Theorem 5.5. With the above notation as $\tau \rightarrow 0$,

$$
Z\left(s, R_{\tau}\right) / \prod_{j=1}^{m} \mathscr{Z}\left(s, \ell_{j}\right) \rightarrow Z\left(s, R_{o}\right)
$$

uniformly on compact subsets of $\left\{\operatorname{Re}\left(s^{2}-s\right)>-\varrho\right\} \cup\{\operatorname{Re} s>1\}$.

Proof. First we consider the case $\operatorname{Re} s>1$. The geodesics of $R_{\tau}$ may be grouped into three types, 1) the pinching geodesics, lengths $\rightarrow 0$; 2) the convergent geodesics, disjoint from the pinching geodesics; and 3) the divergent geodesics, intersecting the 
pinching geodesics. By the Bers result of Sect. 2.5 the lengths of the type 2 geodesics converge to their limiting value. Now since the width of a collar diverges as $\ell \rightarrow 0$, the type 3 geodesics have lengths tending to infinity. For $\operatorname{Re} s>1$ Theorem 5.2 provides a bound on $Z / \prod_{j} \mathscr{Z}\left(\ell_{j}\right)$, and thus we can take the $\tau$-limit under the product to obtain the desired conclusion.

Finally for $1 \geqq \operatorname{Re}\left(s^{2}-s\right)>-\varrho$ the argument is based on the analytic continuation of formula (5.1). Since a bounded sequence of analytic functions contains a convergent subsequence and $Z / \prod_{j} \mathscr{Z}\left(\ell_{j}\right)$ has $Z\left(R_{o}\right)$ as limit for $\operatorname{Re} s>1$, it is enough to bound the quotient. Now from (5.1) and the proof of Theorem 5.3 we see that $Z_{* *}(s)$ and $Q(s)$ are uniformly bounded on compact subsets of $\left\{\operatorname{Re}\left(s^{2}-s\right)>-\varrho\right\}$. Since $Z_{* *} Q=Z / \prod_{j} \mathscr{Z}\left(\ell_{j}\right)$ the conclusion follows.

Remark. An immediate consequence of the considerations of Theorem 5.2 and 5.5 is that the type 3 geodesics are negligible in the limit of $Z\left(s, R_{\tau}\right), \operatorname{Re} s>1$.

\section{Asymptotics of the Bosonic Polyakov $(d=26)$ Integral}

6.1. That the bosonic Polyakov integral diverges will be an easy consequence of Theorems 5.2 and 5.3, and the formula for the Weil-Petersson measure in FenchelNielsen coordinates [33]. In Sect. 6.2 we recall the results of D'Hoker-Phong and take the opportunity to compare our estimate to that of Belavin-Knizhnik. A review of the Fenchel-Nielsen description of the divisor $\mathscr{D}$ is given in the next section $[1,33]$. In the last section we combine our results and discuss the Polyakov integral.

6.2. D'Hoker and Phong find the formula

$$
Z_{g}=\int_{\mathscr{M}_{g}}\left(\operatorname{det} P_{1}^{\dagger} P_{1}\right)^{1 / 2}\left(\frac{2 \pi \operatorname{det}^{\prime} \Delta}{\text { Area }}\right)^{-13} d \mathrm{~W}-\mathrm{P}
$$

for the genus $g$ contribution to the Polyakov partition function ([9], formula (3.15)). Recall that $P_{1}$ is a first order operator mapping vector fields to symmetric traceless 2-tensors, $P_{1}^{\dagger}$ its adjoint, and $\operatorname{det}^{\prime} \Delta$ the functional determinant, zero mode removed, for the Laplacian on functions; $d \mathrm{~W}-\mathrm{P}$ the Weil-Petersson volume form. They continue the calculation and write the integrand as

$$
e^{c(2 g-2)} Z^{\prime}(1)^{-13} Z(2) d \mathrm{~W}-\mathrm{P},
$$

where $c$ is an absolute constant, [9]. By Lemma 5.1, Theorems 5.2 and 5.3 we have that $Z^{\prime}(1)^{-13} Z(2)$ is bounded above and below by multiples (depending only on the genus) of

$$
E=\prod_{j=1}^{m} \exp \left(4 \pi^{2} / \ell_{j}\right) \ell_{j}^{10}\left(\prod_{0<\kappa_{n}<\frac{1}{4}} \kappa_{n}\right)^{-13} .
$$

Now we would like to compare this to the Belavin and Knizhnik result [5]. To do this we must introduce a holomorphic coordinate $t$ (a transverse to the com- 
pactification divisor $\mathscr{D}$, see Sect. 2.5) for describing the degeneration. The change of variables is considered in Sect. 2.2 and also Lemma 2.1. Briefly put

and thus

$$
\left|t_{j}\right| \sim \exp \left(-2 \pi^{2} / \ell_{j}\right)
$$

$$
E(t) \approx \prod_{j=1}^{m}\left|t_{j}\right|^{-2} \times(\text { lower order terms }),
$$

and of course Masur's result (Sect. 2.3) is

$$
d \mathrm{~W}-\mathrm{P} \approx \prod_{j=1}^{m} \frac{\left|d t_{j}\right|^{2}}{\left|t_{j}\right|^{2}\left(\log 1 /\left|t_{j}\right|\right)^{3}} .
$$

In summary the Polyakov integrand behaves as

$$
\prod_{j=1}^{m}\left|t_{j}\right|^{-4} \times(\text { lower order terms }),
$$

where the lower order terms are at most of order $\left(\log \left|t_{j}\right|\right)^{k}$, for some $k$. Lemma 2.1 is not sufficient to obtain the lower order expansion. Our expansion agrees with the Belavin-Knizhnik double-pole result, [5]. A lower order term, due to the norm of the zero modes, does in fact appear for their expansion [39].

6.3. Now we shall review the basics of the description of $\overline{\mathscr{M}}_{g}$ in Fenchel-Nielsen $(\mathrm{F}-\mathrm{N})$ coordinates [1]. As a warning we recall that the change of variables from complex coordinates to $\mathrm{F}-\mathrm{N}$ coordinates is not smooth along $\mathscr{D}$. In brief the relation between $\sigma$, the complex coordinate for opening a node (Sect. 2.5) and $\ell$, the F-N coordinate for opening a node is $|\sigma| \sim \exp \left(-2 \pi^{2} / \ell\right)$. Thus it is possible (and actually happens) that the Weil-Petersson measure on $\overline{\mathscr{M}}_{g}$ is smooth in F-N coordinates and singular in $\mathbb{C}$-coordinates. Of course it is a classical result that the change of variables is smooth on $\mathscr{M}_{\boldsymbol{g}}$.

$\overline{\mathscr{M}}_{g}$ is a compact $V$-manifold in F-N coordinates [33]. Each point has a neighborhood which has a finite cover that is isomorphic to an open set in $\mathbb{R}^{n}$. It is enough for our purposes to describe how a surface is constructed from length-twist parameters $\left(\ell_{j}, \tau_{j}\right) \ell_{j} \geqq 0, \tau_{j} \in \mathbb{R}, j=1, \ldots, 3 g-3$ and a pattern (a partition).

The first observation is that the lengths $\lambda_{1}, \lambda_{2}, \lambda_{3}$ of the boundaries of a pair of pants (pants $=$ three holed sphere with hyperbolic metric and geodesic boundaries) can be arbitrarily prescribed in $[0, \infty)$. A length $\lambda=0$ corresponds to a cusp. Given pants, $P_{1}$ and $P_{2}$, not necessarily distinct, with boundaries $b_{1}$ on $P_{1}$ and $b_{2}$ on $P_{2}$ of the same length, we can form the geometric connected sum $P_{1} \vee P_{2}$ by identifying $b_{1}$ and $b_{2}$ via an isometry. The fundamental observation is that $P_{1} \vee P_{2}$ has a hyperbolic metric whose restrictions to $P_{1}$ and $P_{2}$ is the original metric. By choosing more pants and repeating this process we can close up the boundaries to obtain either a compact surface (all lengths positive) or a surface with nodes. Boundaries of length 0 are formally paired, even though no points are actually identified.

To describe a coordinate neighborhood for $\overline{\mathscr{M}}_{g}$ start with a surface $R$ of genus $g$ with $m$ nodes (see Sect. 2.5). $R_{o}=R$ - \{nodes $\}$ has a hyperbolic metric and we choose $3 g-3-m$ disjoint simple geodesics $\left\{\ell_{j}\right\}$ on $R_{o}$. The topological data $\left\{\gamma_{j}\right\} \cup\{$ nodes $\}$ is our combinatorial pattern (a node is thought of as a geodesic of 
length 0$) . R-\left\{\gamma_{j}\right\} \cup\{$ nodes $\}$ is a union of pants. Now we wish to identify the continuous parameters determining $R$. Each $\gamma_{j}$ has a length $\ell_{j}$ and for each node obviously $\ell_{k}=0$. Now provided $\ell_{j} \neq 0$, we also include the combinatorial data of choosing one other boundary in each pants adjoining $\gamma_{j}$. Dropping perpendiculars from these other boundaries to $\gamma_{j}$ we obtain two distinguished points, one on each side of $\gamma_{j}$. Let $\tau_{j}$ be the distance between these points and $\theta_{j}=2 \pi \tau_{j} / \ell_{j}$, the $\theta_{j}$ are the twist angles. Just as with polar coordinates, $\theta_{k}$ is undefined if $\ell_{k}=0$. Now the tuple $\left(\ell_{j}, \theta_{j}\right)$ provide (polar) coordinates for the local manifold cover of a neighborhood of $R \in \bar{M}_{g}$. The W-P volume form is very simple in these coordinates

$$
d \mathrm{~W}-\mathrm{P}=(2 \pi)^{3-3 g} \prod_{j=1}^{3 g-3} \ell_{j} d \theta_{j} \wedge d \ell_{j}
$$

The simple change of variables $\ell_{j}=2 \pi^{2} /(\log 1 /|t|), \theta_{j}=\operatorname{Im} \log t_{j}$ will carry this formula into Masur's.

The strata of $\mathscr{D}$ are classified by the pattern of the nodes on $R$. For instance the generic surface of $\mathscr{D}$ has one node for which there are $[g / 2]$ patterns; topologically the position of the node is determined by the genera of the components of $R-\{$ nodes $\}$. Similarly a surface with two nodes represents a self intersection point of $\mathscr{D}$.

6.4. Our plan is to give a general method to estimate integrals over $\mathscr{M}_{g}$. For $\beta$, a small constant, we define the $\beta$-thick part of $\mathscr{M}_{g}$ as the compact set of surfaces with all geodesics of length $\geqq \beta$. We shall describe how the complement, $\mathscr{M}_{g}$ ( $\beta$-thin), can be expressed as a union (not disjoint) of sets, each fibered over an open strata of the divisor $\mathscr{D}$.

The combinatorics of the $\mathscr{D}$ strata can be described in terms of partitions and subpartitions of a fixed surface $M$. A subpartition $\mathscr{S} \mathscr{P}=\left\{\left[\gamma_{k}\right]\right\}_{k=1}^{m}$ is a set of free homotopy classes of simple loops, where the loops are neither null nor mutually homotopic. A subpartition of maximal size, a partition, has $3 g-3$ elements. Given a surface $R$ with nodes we can find a map $h: M \rightarrow R$ which collapses a finite disjoint union of simple loops to points and is otherwise a homeomorphism. In this way a surface with nodes determines a subpartition of $M$ : the curves collapsed by $h$. A basic result is that the open strata of $\mathscr{D}$ are in one to one correspondence with the equivalence classes of subpartitions of $M$ and the equivalence is by the homeomorphisms of $M,[1,33]$. Keep in mind that there are a finite number of strata.

An important observation is that there exists a constant $\eta=\eta(g)$ such that each hyperbolic surface has a partition by geodesics of length $\leqq \eta$, [6]. Now for $\alpha<\beta<\eta$ we define $U(\mathscr{S} \mathscr{P}) \subset \mathscr{M}_{g}, \mathscr{S} \mathscr{P}$ as above, to be the subset of surfaces $R$ with a partition $\mathscr{P}=\left\{\left[\delta_{k}\right]\right\}_{k=1}^{3 g-3}$ by geodesics such that: there exists a homeomorphism $f: M \rightarrow R$ with $\left[f\left(\gamma_{k}\right)\right]=\left[\delta_{k}\right]$, and $\ell\left(\delta_{k}\right) \leqq \beta$ for $1 \leqq k \leqq m$ and $\alpha<\ell\left(\delta_{k}\right)<\eta$ for $m+1 \leqq k \leqq 3 g-3$. Roughly speaking $U(\mathscr{S} \mathscr{P})$ is the subset of surfaces near the $\mathscr{D}$-strata corresponding to $\mathscr{S} \mathscr{P}$.

Let $\mathscr{S} \mathscr{P}_{1}, \ldots, \mathscr{S} \mathscr{P}_{p}$ be representatives for the distinct equivalence classes of subpartitions on $M$. We summarize the above discussion with the following.

Lemma 6.1. $\mathscr{M}_{g}(\beta$-thin $)=\bigcup_{j=1}^{p} U\left(\mathscr{S} \mathscr{P}_{j}\right)$. 
Theorem 6.2. The bosonic Polyakov integral $(d=26)$ is infinite.

Proof. It will suffice to estimate the integral for a particular $U(\mathscr{S} \mathscr{P})=\left\{\left(\theta_{j}, \ell_{j}\right): 0\right.$ $<\ell\left(\delta_{j}\right)<\beta$, for $j=1, \ldots, m, \alpha<\ell\left(\delta_{j}\right)<\eta$, for $j=m+1, \ldots, 3 g-3$ and $0 \leqq \theta_{j} \leqq 2 \pi$, all $j\}$. From Sects. 6.2 and 6.3 we have that the Polyakov integrand is bounded below (and above) by

$$
E d \mathrm{~W}-\mathrm{P}=\prod_{j=1}^{m} \exp \left(4 \pi^{2} / \ell_{j}\right) \ell_{j}^{10} \prod_{0<\kappa_{n}<\frac{1}{4}} \kappa_{n}^{-13} d \mathrm{~W}-\mathrm{P} .
$$

The magnitude of the eigenvalue product can be determined from the combinatorics of $\mathscr{S} \mathscr{P}$, using Theorem 3.1. This is not necessary for the present purpose : all we need is that the product is bounded below, a trivial observation. Thus a lower bound for $E d \mathrm{~W}-\mathrm{P}$ is the integrand

$$
\prod_{j=1}^{m} \exp \left(4 \pi^{2} / \ell_{j}\right) \ell_{j}^{10} \prod_{j=1}^{3 g-3} \ell_{j} d \theta_{j} \wedge d \ell_{j} .
$$

Its integral over $U(\mathscr{S P P})$ is a product; the first $m$ length integrals are $\int_{0}^{\beta} \exp \left(4 \pi^{2} / \ell_{j}\right) \ell_{j}^{11} d \ell_{j}$, each infinite. The bosonic Polyakov integral is divergent.

Acknowledgements. It is my pleasure to thank Eric D'Hoker, Charles Epstein, Dorian Goldfeld, Dennis Hejhal and Philip Nelson for their advice and suggestions. I would like to particularly thank D. H. Phong for a great deal of advice, encouragement as well as an introduction to string theory.

\section{References}

1. Abikoff, W.: Topics in the real analytic theory of Teichmüller space. Lecture Notes in Mathematics, Vol. 820. Berlin, Heidelberg, New York: Springer 1980

2. Ahlfors, L.V.: Some remarks on Teichmüller's space of Riemann surfaces. Ann. Math. 74, 171-191 (1961)

3. Ahlfors, L.V.: Conformal invariants. New York: McGraw-Hill 1973

4. Alvarez, O.: Theory of strings with boundaries. Nucl. Phys. B216, 125-184 (1983)

5. Belavin, A.A., Knizhnik, V.G.: Algebraic geometry and the geometry of quantum strings. Phys. Lett. B168, 201 (1986)

6. Bers, L.: Spaces of degenerating Riemann surfaces. Ann. Math. Stud. 79, 43-55 (1974)

7. Buser, P.: Riemannsche Flächen mit Eigenwerten in $\left(0, \frac{1}{4}\right)$. Comment. Math. Helv. 52, 25-34 (1977)

8. Cheng, S.Y.: Eigenvalue comparison theorems and its geometric applications. Math. Z. 143, 289-297 (1975)

9. D'Hoker, E., Phong, D.H.: Multiloop amplitudes for the bosonic Polyakov string. Nucl. Phys. B269, 205-234 (1986)

10. D'Hoker, E., Phong, D.H.: On determinants of Laplacians on Riemann surfaces. Commun. Math. Phys. 105, 537 (1986)

11. Dodzuik, J., Pignataro, T., Randol, B., Sullivan, D.: Estimating small eigenvalues on Riemann surfaces. Preprint

12. Earle, C., Marden, A.: Manuscript in preparation

13. Fay, J.: Analytic torsion and Prym differentials. Ann. Math. Stud. 97, 107-122 (1981) 
14. Fay, J.: Fourier coefficients of the resolvent for a Fuchsian group. J. Reine Angew. Math. 293, 143-203 (1977)

15. Fried, D.: Analytic torsion and closed geodesics on hyperbolic manifolds. Preprint

16. Friedan, D.: Introduction to Polyakov's string theory. In: Les Houches, XXXIX, 1982: Recent advances in field theory and statistical mechanics. New York: Elsevier North Holland 1984

17. Gava, E., Iengo, R., Jayaraman, T., Ramachandran, R.: Multiloop divergences in the closed bosonic string theory. Preprint

18. Hejhal, D.A.: The Selberg trace formula and the Riemann zeta function. Duke Math. J. 43, 441-482 (1976)

19. Hejhal, D.A.: A continuity method for spectral theory on Fuchsian groups. In: Modular forms. Rankins, R.A. (ed.). Ellis-Horwood (1984) pp. 107-140

20. Hejhal, D.A.: Manuscript in preparation

21. Kierlanczyk, M.: Determinants of Laplacians. M.I.T. Thesis, 1986

22. Masur, H.: The extension of the Weil-Petersson metric to the boundary of Teichmüller space. Duke Math. J. 43, 623-635 (1976)

23. Matelski, J.P.: A compactness theorem for Fuchsian groups of the second kind. Duke Math. J. 43, 829-840 (1976)

24. McKean, H.P.: Selberg's trace formula as applied to a compact Riemann surface. Commun. Pure Appl. Math. 25, 225-246 (1972)

25. Mumford, D. : A remark on Mahler's compactness theorem. Proc. A.M.S. 28, 289-294 (1971)

26. Polyakov, A.M.: Quantum geometry of bosonic strings. Phys. Lett. 103B, 207 (1981)

27. Randol, B.: Cylinders in Riemann surfaces. Comment. Math. Helv. 54, 1-5 (1979)

28. Ray, D.B., Singer, I.M. : Analytic torsion for complex manifolds. Ann. Math. 98, 154-177 (1973)

29. Sarnak, P.: Determinants of Laplacians. Commun. Math. Phys. 110, 113-120 (1987)

30. Schoen, R., Wolpert, S., Yau, S.T.: Geometric bounds on the low eigenvalues of a compact surface. Proc. Symp. Pure Math. 36, 279-285 (1980)

31. Weinberger, H.: Variational methods for eigenvalue approximation. Philadelphia, PA: Soc. Ind. Appl. Math. 1974

32. Wolpert, S.A.: On the symplectic geometry of deformations of a hyperbolic surface. Ann. Math. 117, 207-234 (1983)

33. Wolpert, S.A.: On the Weil-Petersson geometry of the moduli space of curves. Am. J. Math. 107, 969-997 (1985)

34. Wolpert, S.A.: Chern forms and the Riemann tensor for the moduli space of curves. Invent. Math. 85, 119 (1986)

35. Ahlfors, L.V.: Lectures on quasiconformal mappings. New York: Van Nostrand 1966

36. Cheng, S.Y., Li, P.: Heat kernel estimates and lower bounds for the eigenvalues. Comment. Math. Helv. 56, 327-338 (1981)

37. Fay, J.D.: Theta functions on Riemann surfaces. Lecture Notes Vol. 352. Berlin, Heidleberg, New York: Springer 1973

38. Hejhal, D.A.: The Selberg trace formula for PSL $(2 ; \mathbb{R})$, Vol. 1. Lecture Notes Vol. 548. Berlin, Heidelberg, New York: Springer 1976

39. Nelson, P.: Lectures on strings and moduli space. Preprint

40. Hejhal, D.A.: The Selberg trace formula for PSL (2; R), Vol. 2. Lecture Notes Vol. 1001. Berlin, Heidelberg, New York: Springer 1983

41. McKean, H.P., Singer, I.M.: Curvature and the eigenvalues of the Laplacian. J. Differ. Geom. 1, 43-69 (1967)

Communicated by S.-T. Yau

Received July 29, 1986; in revised form March 13, 1987 
